\title{
Squeezing and resonance in a generalized Caldirola-Kanai type quantum parametric oscillator
}

\author{
Şirin A. Büyükaşık \\ Department of Mathematics, Izmir Institute of Technology, 35430 Urla, Izmir, Turkey
}

(Received 30 August 2017; accepted 9 July 2018; published online 7 August 2018)

\begin{abstract}
The evolution operator of a Caldirola-Kanai type quantum parametric oscillator with a generalized quadratic Hamiltonian is obtained using the Wei-Norman Lie algebraic approach, and time evolution of the eigenstates of a harmonic oscillator and Glauber coherent states is found explicitly. Behavior of this oscillator is investigated under the influence of the external mixed term $B(t)(\hat{q} \hat{p}+\hat{p} \hat{q}) / 2$, which affects the squeezing properties of the wave packets, and linear terms $D_{0}(t) \hat{q}, E_{0}(t) \hat{p}$ responsible for their displacement in time. According to this, we construct all exact quantum models with different parameters $B(t)$, for which the structure of the Caldirola-Kanai oscillator in position space is preserved. Then, for each model, we obtain explicit solutions and analyze the squeezing and displacement properties of the wave packets according to the frequency modification by $B(t)$ and periodic forces in the corresponding classical equation of motion. Published by AIP Publishing. https://doi.org/10.1063/1.5002186
\end{abstract}

\section{INTRODUCTION}

Quantum harmonic oscillators with explicitly time-dependent Hamiltonians appear in many physical branches such as quantum optics, ${ }^{1-3}$ quantum fluid dynamics, ${ }^{4}$ ion-traps,${ }^{5}$ cosmology, ${ }^{6}$ quantum information, ${ }^{7}$ and quantum computation. ${ }^{8}$ The best known model of the quantum parametric oscillator is the Caldirola-Kanai (CK) oscillator with Hamiltonian

$$
\hat{H}(t)=\frac{e^{-\gamma t}}{2 m} \hat{p}^{2}+\frac{1}{2} m \omega_{0}^{2} e^{\gamma t} \hat{q}^{2}
$$

having exponentially increasing mass $\mu(t)=m e^{\gamma t}$, which was introduced by Caldirola ${ }^{9}$ and Kanai $^{10}$ as an attempt to describe dissipation in quantum systems. ${ }^{11}$ Since then, many different approaches were used to obtain exact solutions and study their properties. Well-known methods are the Husimi ansatz, ${ }^{12}$ the path integral, ${ }^{13}$ the Lewis-Riesenfeld invariant, ${ }^{14}$ the WeiNorman dynamical symmetry, ${ }^{15}$ and canonical transformations to the corresponding constant mass problem. ${ }^{16,17}$ These methods are often applied also to the generalized quadratic oscillators and in particular to the driven Caldirola-Kanai oscillator, which is extensively studied by many authors. ${ }^{18-27}$

In the present work, we consider a generalized Caldirola-Kanai oscillator, by adding to Eq. (1) the mixed term $B(t)(\hat{q} \hat{p}+\hat{p} \hat{q}) / 2$, and the linear terms $D_{0}(t) \hat{q}, E_{0}(t) \hat{p}$, with time-dependent squeezing parameter $B(t)$ and displacement parameters $D_{0}(t), E_{0}(t)$. Then, the evolution operator for the generalized problem is obtained using the Wei-Norman algebraic approach, ${ }^{28}$ which allows us to find explicitly the time-evolution of given initial states. The main goal of this work is to study the squeezing and displacement properties of the CK-oscillator under the influence of the external terms and the corresponding time-dependent parameters.

As known, coherent and squeezed states in quantum mechanics were discovered almost a century ago and then rediscovered by many authors. ${ }^{29-38}$ Coherent states are known as minimum uncertainty states for which $\Delta \hat{q}=\Delta \hat{p}$, while squeezed states are generalization of the coherent states, which in simplest case obey the minimum uncertainty principle, but have less uncertainty in one quadrature at the expense of increased uncertainty in the other. Many of the properties of the squeezed states (two-photon coherent states) were investigated by Yuen. ${ }^{39}$ Today, it is known that they have 
exact realization and application in many physical areas, such as quantum optics and quantum electrodynamics. ${ }^{39-42}$

For standard harmonic oscillator (SHO), with $\hat{a}$ and $\hat{a}^{\dagger}$ being the annihilation and creation operators, respectively, coherent states and squeezed states can be defined in different but equivalent ways. One way to obtain coherent states is to apply the displacement operator $D(\alpha)=\exp \left[\alpha \hat{a}^{\dagger}-\alpha^{*} \hat{a}\right]$ to the ground state. Then, squeezed coherent states can be found by applying the squeeze operator $\hat{S}(z)=\exp \left[\frac{1}{2}\left(z \hat{a}^{\dagger 2}-z^{*} \hat{a}^{2}\right]\right.$ to the coherent states. In Ref. 43, it was shown that for SHO a simple way of realizing the displaced and squeezed number states is to add to $\hat{H}_{0}$, at some moment of time, a term of the form $\frac{1}{2} \omega_{1}^{2} q^{2}-f_{0} q$, which corresponds to the displacement of the oscillator and changing its frequency.

On the other side, when the Hamiltonian $\hat{H}(t)$ depends explicitly on time, like in Eq. (1), squeezing affects appear naturally due to the time variable parameters. ${ }^{36,43-45}$ Moreover, for a general quadratic oscillator, it is known that parameter $B(t)$ effects both the squeezing and displacement properties, while the driving linear forces and their parameters $D_{0}(t)$ and $E_{0}(t)$ contribute only to the displacement of the wave packets. ${ }^{23,28,43,44}$ According to this, choosing periodic driving forces, resonance in coherent states of a quantum damped oscillator was addressed long time ago in Ref. 18. Then, in Ref. 23, coherent states of a general time-dependent harmonic oscillator were obtained using the LewisRiesenfeld approach, and it was shown that displaced wave packets for the CK-oscillator in coherent states oscillate back and forth with time about the center as for the classical oscillator. Then, many illustrative examples including the behavior at resonance frequency were constructed. However, all of these examples were investigated when the squeezing parameter is zero, that is, $B(t)=0$. Recently, squeezing effects due to certain non-zero parameters $B(t)$ were discussed in Ref. 46 but using the Heisenberg formalism.

In this work, we solve a generalized Caldirola-Kanai oscillator by the evolution operator method and investigate the possibility to control squeezing of the wave packets and their displacement at resonance frequency, by changing the squeezing parameter $B(t)$, when preserving the structure of the original CK-oscillator. For this, in Sec. II, we first write the evolution operator for the generalized CK-oscillator, and using it we obtain the wave functions, probability densities, expectation values, and uncertainties. In Sec. III, we find all possible parameters $B(t)$, for which the corresponding classical equation in position space has constant frequency and damping. It happens that $B(t)$ should satisfy a Riccati equation with constant coefficients, and therefore it could be any real constant, as well as it could be a function of time. Then, for every possible $B(t)$, we construct the Caldirola-Kanai quantum models and study them separately. More precisely, in Sec. III, to be able to compare all models and see the differences between them, we provide some basic estimates for the standard Caldirola-Kanai model $(B(t)=0)$, and the models with constant parameter $B(t)=B_{0}$. Then, in Sec. IV, we investigate three CK-models with different time-dependent parameters $B(t)$. For each model, we derive the squeezing coefficient and uncertainties and discuss their properties according to the frequency modification in both position space and momentum space. In addition to this, we introduce simple sinusoidal driving forces and study the resonance phenomena for the expectation values at time-evolved wave functions. Many illustrative figures are constructed. Finally, Sec. V includes the brief summary and discussion of the present results.

\section{SOLUTION OF THE GENERALIZED CALDIROLA-KANAI OSCILLATOR}

Consider the evolution problem for quantum harmonic oscillators

$$
\begin{gathered}
i \hbar \frac{\partial}{\partial t} \Psi(q, t)=\hat{H}_{g}(t) \Psi(q, t), \\
\Psi\left(q, t_{0}\right)=\Psi_{0}(q), \quad-\infty<q<\infty,
\end{gathered}
$$

with a general quadratic Hamiltonian

$$
\hat{H}_{g}(t)=\frac{e^{-\gamma t}}{2} \hat{p}^{2}+\frac{1}{2} \omega_{0}^{2} e^{\gamma t} \hat{q}^{2}+\frac{B(t)}{2}(\hat{q} \hat{p}+\hat{p} \hat{q})-e^{\gamma t} D(t) \hat{q}+e^{-\gamma t} E(t) \hat{p},
$$


where $\mu(t)=m e^{\gamma t}, \gamma>0, m=1$, is the exponentially increasing mass, $B(t), D(t), E(t)$ are the arbitrary real-valued parameters depending on time, and $\hat{q}=q, \hat{p}=-i \hbar \partial / \partial q$. Since the Hamiltonian is a linear combination of Heisenberg-Weyl and $s u(1,1)$ Lie algebra generators,

$$
\begin{gathered}
\hat{E}_{1}=i q, \quad \hat{E}_{2}=\frac{\partial}{\partial q}, \quad \hat{E}_{3}=i \hat{I}, \\
\hat{K}_{-}=-\frac{i}{2} \frac{\partial^{2}}{\partial q^{2}}, \quad \hat{K}_{+}=\frac{i}{2} q^{2}, \quad \hat{K}_{0}=\frac{1}{2}\left(q \frac{\partial}{\partial q}+\frac{1}{2}\right),
\end{gathered}
$$

in our previous work, ${ }^{28}$ using the Wei-Norman Lie algebraic approach the evolution operator was found as

$$
\begin{aligned}
\hat{U}_{g}\left(t, t_{0}\right)= & \exp \left(\frac{i}{\hbar} \int_{t_{0}}^{t}\left[\frac{-1}{2 \mu(s)} p_{p}^{2}(s)-E(s) p_{p}(s)+\frac{\mu(s) \omega^{2}(s)}{2} x_{p}^{2}(s)\right] d s\right) \\
& \times \exp \left(i p_{p}(t) q\right) \times \exp \left(-x_{p}(t) \frac{\partial}{\partial q}\right) \times \exp \left(i \frac{\mu(t)}{2 \hbar}\left(\frac{\dot{x}_{1}(t)}{x_{1}(t)}-B(t)\right) q^{2}\right) \\
& \left.\times \exp \left(\ln \mid \frac{x_{1}\left(t_{0}\right)}{x_{1}(t)}\right)\left(q \frac{\partial}{\partial q}+\frac{1}{2}\right)\right) \times \exp \left(\frac{i}{2} \hbar x_{1}^{2}\left(t_{0}\right)\left(\frac{x_{2}(t)}{x_{1}(t)}\right) \frac{\partial^{2}}{\partial q^{2}}\right),
\end{aligned}
$$

where $x_{1}(t), x_{2}(t)$ are independent homogeneous solutions of the corresponding classical equation of motion,

$$
\ddot{x}+\gamma \dot{x}+\left(\omega_{0}^{2}-\left(\dot{B}+B^{2}+\gamma B\right)\right) x=D+e^{-\gamma t}(\dot{E}+B E),
$$

satisfying the initial conditions $x_{1}(0)=x_{0} \neq 0, \dot{x}_{1}(0)=x_{0} B(0)$, and $x_{2}(0)=0, \dot{x}_{2}(0)=1 / x_{0}$, and $x_{p}(t)$ is a particular solution of (6) satisfying $x_{p}(0)=0, \dot{x}_{p}(0)=E(0)$. Also, one has the classical oscillator in momentum space

$$
\ddot{p}-\gamma \dot{p}+\left(\omega_{0}^{2}+\left(\dot{B}-B^{2}-\gamma B\right)\right) p=e^{\gamma t}(\dot{D}-B D)-\omega_{0}^{2} E,
$$

with independent homogeneous solutions of the form

$$
p_{1}(t)=e^{\gamma t}\left(\dot{x}_{1}(t)-B(t) x_{1}(t)\right), \quad p_{2}(t)=e^{\gamma t}\left(\dot{x}_{2}(t)-B(t) x_{2}(t)\right)
$$

and a particular solution $p_{p}(t)=e^{\gamma t}\left(\dot{x}_{p}(t)-B(t) x_{p}(t)-e^{-\gamma t} E(t)\right)$.

Knowing the evolution operator, we obtain the exact time evolution of the eigenstates and coherent states of the harmonic oscillator.

\section{A. Time-evolution of the eigenstates of the harmonic oscillator}

First, as initial functions of the quantum problem (2) and (3), we choose the normalized eigenstates of the standard Hamiltonian,

$$
\varphi_{k}(q)=N_{k} e^{-\frac{\omega_{0}}{2 \hbar} q^{2}} H_{k}\left(\sqrt{\frac{\omega_{0}}{\hbar}} q\right),
$$

where $H_{k}\left(\sqrt{\omega_{0} / \hbar} q\right)$ are Hermite polynomials, $N_{k}=\left(2^{k} k !\right)^{-1 / 2}\left(\omega_{0} / \pi \hbar\right)^{1 / 4}$ are normalization constants, and eigenvalues are $E_{k}=\left(\hbar \omega_{0}\right)(k+1 / 2), k=0,1,2, \ldots$ Then, time-evolved wave functions are of the form

$$
\begin{aligned}
& \Psi_{k}(q, t)=N_{k} \sqrt{R_{B}(t)} \times \exp \left[i\left(k+\frac{1}{2}\right) \arctan \left(-\omega_{0} x_{1}^{2}\left(t_{0}\right)\left(\frac{x_{2}(t)}{x_{1}(t)}\right)\right)\right] \\
& \times \exp \left[-\frac{i}{2 \hbar} e^{\gamma t}\left(B(t)+\frac{\dot{R}_{B}(t)}{R_{B}(t)}\right)\left(q-x_{p}(t)\right)^{2}+\frac{i}{\hbar}\left(e^{\gamma t}\left(\dot{x}_{p}(t)-B(t) x_{p}(t)-e^{-\gamma t} E(t)\right)\right) q\right] \\
& \times \exp \left[\frac{-i}{2 \hbar} \int_{t_{0}}^{t} e^{\gamma s}\left[\dot{x}_{p}^{2}(s)-2 B(s) x_{p}(s) \dot{x}_{p}(s)+\left(B^{2}(s)-\omega_{0}^{2}\right) x_{p}^{2}(s)-e^{-2 \gamma s} E^{2}(s)\right] d s\right] \\
& \times \exp \left[-\frac{1}{2}\left(\sqrt{\frac{\omega_{0}}{\hbar}} R_{B}(t)\left(q-x_{p}(t)\right)\right)^{2}\right] \times H_{k}\left(\sqrt{\frac{\omega_{0}}{\hbar}} R_{B}(t)\left(q-x_{p}(t)\right)\right),
\end{aligned}
$$


and probability densities become

$$
\rho_{k}(q, t)=N_{k}^{2} R_{B}(t) \exp \left(-\left(\sqrt{\frac{\omega_{0}}{\hbar}} R_{B}(t)\left(q-x_{p}(t)\right)\right)^{2}\right) \times H_{k}^{2}\left(\sqrt{\frac{\omega_{0}}{\hbar}} R_{B}(t)\left(q-x_{p}(t)\right)\right),
$$

where

$$
R_{B}(t)=\sqrt{\frac{x_{0}^{2}}{x_{1}^{2}(t)+\left(\omega_{0} x_{0}^{2} x_{2}(t)\right)^{2}}}
$$

is the squeezing coefficient determined by $x_{1}(t), x_{2}(t)$, and $x_{p}(t)$ is the displacement of the wave packets. Thus, $R_{B}(t)$ is responsible for the amplitude and width properties of the wave packets, while $x_{p}(t)$ shows how their center moves with time. We note that the squeezing coefficient $R_{B}(t)$ depends on the squeezing parameter $B(t)$ but does not depend on the external term parameters $D(t)$ and $E(t)$. On the other hand, the displacement $x_{p}(t)$ depends on all parameters of the Hamiltonian. We note also that $\Psi_{k}(q, t)$ and $\rho_{k}(q, t)$ have moving zeros due to the zeros of the Hermite polynomials. Precisely, if $\tau_{k}^{(l)}, l=1,2, \ldots k$, are the zeros of the Hermite polynomial $H_{k}(\xi)$, i.e., $H_{k}\left(\tau_{k}^{(l)}\right)=0$, then for each $k=1,2,3, \ldots$, the motion of the zeros is given by

$$
q_{k}^{(l)}(t)=\sqrt{\frac{\hbar}{\omega_{0}}} \frac{\tau_{k}^{(l)}}{R_{B}(t)}+x_{p}(t), \quad l=1,2, \ldots, k
$$

Next, it is easy to see that the expectation values at wave functions $\Psi_{k}(q, t)$ are

$$
\langle\hat{q}\rangle_{k}(t)=x_{p}(t), \quad\langle\hat{p}\rangle_{k}(t)=p_{p}(t),
$$

and therefore the expectation values of squares of position and momentum become

$$
\begin{gathered}
\left\langle\hat{q}^{2}\right\rangle_{k}(t)=\hbar\left(k+\frac{1}{2}\right)\left(\frac{1}{\omega_{0} R_{B}^{2}(t)}\right)+x_{p}^{2}(t), \\
\left\langle\hat{p}^{2}\right\rangle_{k}(t)=\hbar\left(k+\frac{1}{2}\right)\left(\omega_{0} R_{B}^{2}(t)\left[1+\frac{e^{2 \gamma t}}{\left(\omega_{0} R_{B}^{2}(t)\right)^{2}}\left(\frac{\dot{R}_{B}(t)}{R_{B}(t)}+B(t)\right)^{2}\right]+p_{p}^{2}(t) .\right.
\end{gathered}
$$

As a result, the uncertainties for $\hat{q}$ and $\hat{p}$ are found as

$$
\begin{aligned}
& (\Delta \hat{q})_{k}(t)=\sqrt{\frac{\hbar}{\omega_{0}}\left(k+\frac{1}{2}\right)} \frac{1}{R_{B}(t)}, \\
& (\Delta \hat{p})_{k}(t)=\sqrt{\omega_{0} \hbar\left(k+\frac{1}{2}\right)} R_{B}(t) \sqrt{1+\frac{e^{2 \gamma t}}{\left(\omega_{0} R_{B}^{2}(t)\right)^{2}}\left(\frac{\dot{R}_{B}(t)}{R_{B}(t)}+B(t)\right)^{2}},
\end{aligned}
$$

and the uncertainty product in state $\Psi_{k}(q, t)$ becomes

$$
(\Delta \hat{q})_{k}(\Delta \hat{p})_{k}(t)=\hbar\left(k+\frac{1}{2}\right) \sqrt{1+\frac{e^{2 \gamma t}}{\left(\omega_{0} R_{B}^{2}(t)\right)^{2}}\left(\frac{\dot{R}_{B}(t)}{R_{B}(t)}+B(t)\right)^{2}}, k=0,1,2, \ldots
$$

\section{B. Time-evolution of coherent states}

Second, as initial states for the evolution problem (2) and (3), we take the coherent states

$$
\phi_{\alpha}^{0}(q)=\left(\frac{\omega_{0}}{\pi \hbar}\right)^{1 / 4} e^{-\frac{i}{2 \hbar}\langle\hat{q}\rangle_{\alpha}\langle\hat{p}\rangle_{\alpha}} e^{\frac{i}{\hbar}\langle\hat{p}\rangle_{\alpha} q} e^{-\frac{\omega_{0}}{2 \hbar}\left(q-\langle\hat{q}\rangle_{\alpha}\right)^{2}},
$$


where $\langle\hat{q}\rangle_{\alpha}=\sqrt{2 \hbar /\left(\omega_{0}\right)} \alpha_{1},\langle\hat{p}\rangle_{\alpha}=\sqrt{2 \omega_{0} \hbar} \alpha_{2}, \alpha=\alpha_{1}+i \alpha_{2}, \alpha_{1}, \alpha_{2}$ are real constants. Applying the evolution operator $\hat{U}_{g}\left(t, t_{0}\right)$, time-evolved coherent states for the generalized oscillator are found explicitly,

$$
\begin{aligned}
\Phi_{\alpha}(q, t)= & \left(\frac{\omega_{0}}{\pi \hbar}\right)^{1 / 4} R_{B}(t) \times \sqrt{\frac{x_{1}(t)}{x_{0}}-i\left(\omega_{0} x_{0}\right) x_{2}(t)} \\
& \times \exp \left[\frac{i}{\hbar} \int_{t_{0}}^{t}\left(\frac{-e^{-\gamma t}}{2} p_{p}^{2}(s)-e^{-\gamma s} E(s) p_{p}(s)+\frac{e^{\gamma t} \omega_{0}^{2}}{2} x_{p}^{2}(s)\right) d s\right] \\
& \times \exp \left[-i \omega_{0} x_{2}(t) R_{B}^{2}(t)\left(x_{1}(t)-i\left(\omega_{0} x_{0}^{2}\right) x_{2}(t)\right) \alpha^{2}+\frac{\alpha^{2}-|\alpha|^{2}}{2}\right] \\
& \times \exp \left(\frac{i}{\hbar} p_{p}(t) q\right) \times \exp \left[\frac{-i}{2 \hbar} e^{\gamma t}\left(B(t)+\frac{\dot{R}_{B}(t)}{R_{B}(t)}\right)\left(q-x_{p}(t)\right)^{2}\right] \\
& \times \exp \left\{-R_{B}^{2}(t)\left[\sqrt{\frac{\omega_{0}}{2 \hbar}}\left(q-x_{p}(t)\right)-\left(\frac{x_{1}(t)}{x_{0}}-i\left(\omega_{0} x_{0}\right) x_{2}(t)\right) \alpha\right]^{2}\right\},
\end{aligned}
$$

and the corresponding probability densities become

$$
\rho_{\alpha}(q, t)=\sqrt{\frac{\omega_{0}}{\pi \hbar}} \times R_{B}(t) \times \exp \left\{-\left(\sqrt{\frac{\omega_{0}}{\hbar}} R_{B}(t)\left(q-\langle\hat{q}\rangle_{\alpha}(t)\right)\right)^{2}\right\},
$$

with squeezing coefficient $R_{B}(t)$ and displacement $\langle\hat{q}\rangle_{\alpha}(t)$, where expectations at $\Phi_{\alpha}(q, t)$ are

$$
\begin{aligned}
& \langle\hat{q}\rangle_{\alpha}(t)=\sqrt{\frac{2 \hbar}{\omega_{0}}}\left(\frac{\alpha_{1}}{x_{0}} x_{1}(t)+\alpha_{2}\left(\omega_{0} x_{0}\right) x_{2}(t)\right)+x_{p}(t), \\
& \langle\hat{p}\rangle_{\alpha}(t)=\sqrt{\frac{2 \hbar}{\omega_{0}}}\left(\frac{\alpha_{1}}{x_{0}} p_{1}(t)+\alpha_{2}\left(\omega_{0} x_{0}\right) p_{2}(t)\right)+p_{p}(t) .
\end{aligned}
$$

Thus, coherent states are Gaussian wave packets, whose center follows the classical trajectory given by $\langle\hat{q}\rangle_{\alpha}(t)$. Next, finding the expectation of squares

$$
\begin{aligned}
\left\langle\hat{q}^{2}\right\rangle_{\alpha}(t)= & {\left[\sqrt{\frac{2 \hbar}{\omega_{0}}}\left(\alpha_{1} \frac{x_{1}(t)}{x_{0}}+\alpha_{2}\left(\omega_{0} x_{0}\right) x_{2}(t)\right)+x_{p}(t)\right]^{2}+\frac{\hbar}{2 \omega_{0} R_{B}^{2}(t)}, } \\
\left\langle\hat{p}^{2}\right\rangle_{\alpha}(t)= & {\left[\sqrt{\frac{2 \hbar}{\omega_{0}}}\left(\frac{\alpha_{1}}{x_{0}} p_{1}(t)+\alpha_{2}\left(\omega_{0} x_{0}\right) p_{2}(t)\right)+p_{p}(t)\right]^{2} } \\
& +\frac{\hbar}{2 \omega_{0}}\left[\left(\omega_{0} R_{B}(t)\right)^{2}+\frac{e^{2 \gamma t}}{R_{B}^{2}(t)}\left(\frac{\dot{R}_{B}(t)}{R_{B}(t)}+B(t)\right)^{2}\right]
\end{aligned}
$$

gives the uncertainties for $\hat{q}$ and $\hat{p}$

$$
\begin{gathered}
(\Delta \hat{q})_{\alpha}(t)=\sqrt{\frac{\hbar}{2 \omega_{0}}} \frac{1}{R_{B}(t)}, \\
(\Delta \hat{p})_{\alpha}(t)=\sqrt{\frac{\omega_{0} \hbar}{2}} R_{B}(t) \sqrt{1+\frac{e^{2 \gamma t}}{\left(\omega_{0} R_{B}^{2}(t)\right)^{2}}\left(\frac{\dot{R}_{B}(t)}{R_{B}(t)}+B(t)\right)^{2}} .
\end{gathered}
$$

It follows that the uncertainty product at time-evolved coherent states for the generalized harmonic oscillator is 


$$
(\Delta \hat{q})_{\alpha}(\Delta \hat{p})_{\alpha}(t)=\frac{\hbar}{2} \sqrt{1+\frac{e^{2 \gamma t}}{\left(\omega_{0} R_{B}^{2}(t)\right)^{2}}\left(\frac{\dot{R}_{B}(t)}{R_{B}(t)}+B(t)\right)^{2}},
$$

where clearly $(\Delta \hat{q})_{\alpha}(\Delta \hat{p})_{\alpha} \geq \hbar / 2$. Even though the uncertainties do not depend on $\alpha$, we shall keep $\alpha$ as a subscript indicating that we consider uncertainties at coherent states.

\section{FREQUENCY MODIFICATION AND CALDIROLA-KANAI MODELS}

We have seen that parameter $B(t)$ modifies the frequency of the classical oscillator (6), and therefore it changes solutions $x_{1}(t), x_{2}(t)$, which determine the squeezing coefficient $R_{B}(t)$. Thus, $B(t)$ has influence on the uncertainties and on the expectation values of the position and momentum as well. Our goal is to investigate the influence of some special choices of $B(t)$ on the behavior of the quantum oscillator. For this, we recall the homogeneous classical equation in position space,

$$
\ddot{x}+\gamma \dot{x}+\left(\omega_{0}^{2}-\left(\dot{B}+B^{2}+\gamma B\right)\right) x=0 .
$$

In general, an arbitrary parameter $B(t)$ can modify the original frequency and change it essentially. Here, to preserve the oscillator structure, we shall choose $B(t)$ to satisfy the equation

$$
-\left(\dot{B}+B^{2}+\gamma B\right)=\Lambda_{0}^{2},
$$

where $\Lambda_{0}^{2}>-\omega_{0}^{2}$. Then, (23) takes the form

$$
\ddot{x}+\gamma \dot{x}+\left(\omega_{0}^{2}+\Lambda_{0}^{2}\right) x=0,
$$

with $\omega_{0}^{2}+\Lambda_{0}^{2}>0, \Lambda_{0}^{2}$ - being the frequency modification in position space, and

$$
\Omega_{d}^{2}=\omega_{0}^{2}+\Lambda_{0}^{2}-\frac{\gamma^{2}}{4}
$$

will give the frequency $\Omega_{d}$ of the modified damped oscillator. The corresponding homogeneous equation for momentum is

$$
\ddot{p}-\gamma \dot{p}+\left(\omega_{0}^{2}+\Lambda_{p}^{2}(t)\right) p=0,
$$

where $\Lambda_{p}^{2}(t) \equiv \dot{B}(t)-B^{2}(t)-\gamma B(t)$ is the modification of the frequency in momentum space.

Thus, the exact form of the oscillators in position and momentum space will be determined by the Riccati equation (24), for which different types of solutions exist according to the sign of $\Omega_{B}^{2}=\Lambda_{0}^{2}-\gamma^{2} / 4$. In Secs. III A, III B and Sec. IV, we investigate these possibilities separately and study their influence on the corresponding quantum evolution problem.

\section{A. Standard Caldirola-Kanai oscillator, $B(t)=0$}

To be able to compare different models, we shortly recall some results for the standard CK-model. That is, first we consider the Hamiltonian $H_{g}(t)$ given by (4), with $B(t)=0$. The corresponding classical equation of motion is

$$
\ddot{x}+\gamma \dot{x}+\omega_{0}^{2} x=D+e^{-\gamma t} \dot{E},
$$

where $\gamma>0$ is the damping coefficient and $\omega_{0}>0$ is the natural frequency, that is, the frequency of the undamped $(\gamma=0)$ oscillator. Then, according to $\Omega_{0}^{2}=\omega_{0}^{2}-\gamma^{2} / 4$, there are three possible cases, $\Omega_{0}^{2}<0, \Omega_{0}^{2}=0, \Omega_{0}^{2}>0$. In this work, we shall treat explicitly only the more interesting case of weak damping.

Underdamping case $(B(t)=0)$ : When $\Omega_{0}^{2}>0$, homogeneous solutions satisfying the required initial conditions $x_{1}(0)=x_{0} \neq 0, \dot{x}_{1}(0)=0$ and $x_{2}(0)=0, \dot{x}_{2}(0)=1 / x_{0}$, are, respectively,

$$
x_{1}(t)=x_{0} \frac{\omega_{0}}{\Omega_{0}} e^{-\frac{\gamma t}{2}} \cos \left(\Omega_{0} t-\delta_{0}\right), \quad x_{2}(t)=\frac{1}{\Omega_{0} x_{0}} e^{-\frac{\gamma t}{2}} \sin \left(\Omega_{0} t\right),
$$


where $\Omega_{0}=\sqrt{\omega_{0}^{2}-\gamma^{2} / 4}$ is the frequency of the damped oscillator and $\delta_{0}=\tan ^{-1}\left(\gamma / 2 \Omega_{0}\right)$ is the phase shift. The classical oscillator in momentum space becomes

$$
\ddot{p}-\gamma \dot{p}+\omega_{0}^{2} p=e^{\gamma t} \dot{D}-\omega_{0}^{2} E,
$$

with independent homogeneous solutions of the form

$$
p_{1}(t)=-x_{0} \frac{\omega_{0}^{2}}{\Omega_{0}} e^{\gamma t / 2} \sin \left(\Omega_{0} t\right), \quad p_{2}(t)=\frac{\omega_{0}}{x_{0} \Omega_{0}} e^{\gamma t / 2} \cos \left(\Omega_{0} t+\delta_{0}\right) .
$$

For the standard CK-oscillator, the squeezing coefficient is

$$
R_{0}(t)=\frac{e^{\gamma t / 2}}{\sqrt{\frac{\omega_{0}^{2}}{\Omega_{0}^{2}}\left[\cos ^{2}\left(\Omega_{0} t-\delta_{0}\right)+\sin ^{2}\left(\Omega_{0} t\right)\right]}},
$$

and it shows that the amplitude and width of the wave packets of the corresponding quantum oscillator are smooth and oscillatory. We note that when the frequency $\omega_{0}$ increases, the amplitude of oscillations of $R_{0}(t)$ decreases. Also, when $\gamma \rightarrow 0$, one has $\left(\omega_{0}^{2} / \Omega_{0}^{2}\right) \rightarrow 1, \delta_{0} \rightarrow 0$ so that $R_{0}(t)$ approaches the critical squeezing $R_{0}(t)=e^{\gamma t / 2}$; see Fig. 1(a). Therefore, knowing $R_{0}(t)$, and formulas (20) and (21) for $\Delta \hat{q}$ and $\Delta \hat{p}$, respectively, shows us that wave packets exhibit oscillatory squeezing in position $q$, which approaches zero at large times, at the expense of oscillatory spreading in momentum $p$; see Fig. 2. As a result, the uncertainty product at coherent states is

$(\Delta \hat{q})_{\alpha}(\Delta \hat{p})_{\alpha}(t)=\frac{\hbar}{2} \sqrt{1+\frac{\omega_{0}^{2}}{\Omega_{0}^{4}}\left[\frac{\gamma}{2}\left[\cos ^{2}\left(\Omega_{0} t-\delta_{0}\right)+\sin ^{2}\left(\Omega_{0} t\right)\right]+\frac{\Omega_{0}}{2}\left[\sin \left(2\left(\Omega_{0} t-\delta_{0}\right)\right)-\sin \left(2 \Omega_{0} t\right)\right]\right]^{2}}$,

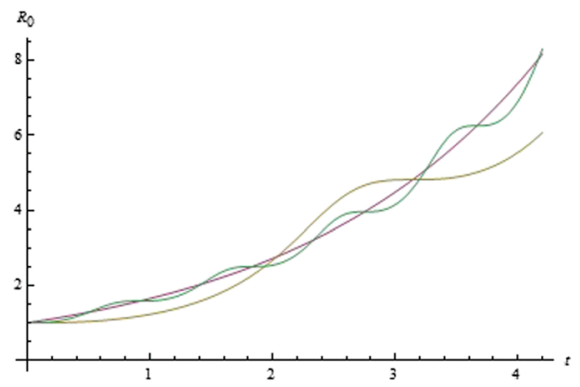

(a)

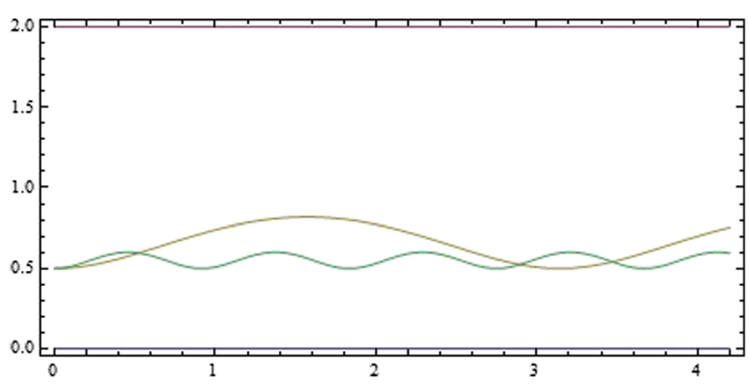

(b)

FIG. 1. Standard CK-model: (a) $R_{0}(t)$ with $\gamma=1$ and $\omega_{0}=\sqrt{5} / 2, \sqrt{12}, \hbar=1$. (b) Uncertainty product $(\Delta \hat{q})_{\alpha}(\Delta \hat{p})_{\alpha}(t)$.

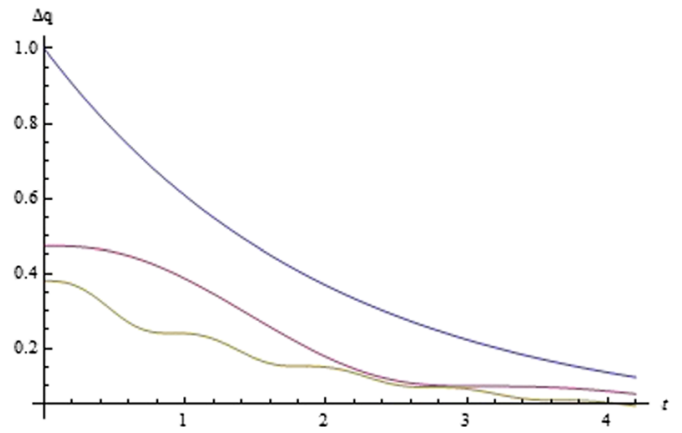

(a)

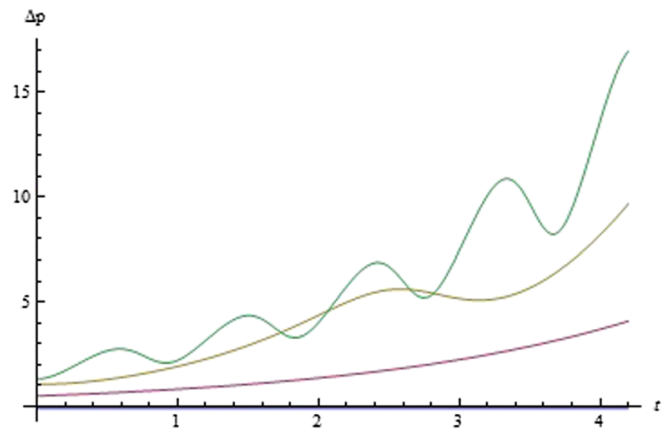

(b)

FIG. 2. Standard CK-model: $\gamma=1$ and $\omega_{0}=\sqrt{5} / 2, \sqrt{12}, \hbar=1$. (a) Uncertainty $\Delta \hat{q}(t)$. (b) Uncertainty $\Delta \hat{p}(t)$. 
which is oscillatory and bounded for all $t \geq 0$, and when $\gamma \rightarrow 0$, it approaches the minimum uncertainty $\Delta \hat{q} \Delta \hat{p}=\hbar / 2$, as shown in Fig. 1(b).

Periodic forces and resonance $(B(t)=0)$ : The total force in Eq. (28) is $F(t)=D+e^{-\gamma t} \dot{E}$, and in general to have sinusoidal type of force, one can consider any $D(t)$ and $E(t)$ such that $F(t)=F_{0} \cos (\omega t$ $\left.+\delta_{d}\right), F_{0}, \omega$, and $\delta_{d}$ arbitrary. For simplicity, we shall assume that $D(t)=D_{0} \cos (\omega t)$, with $D_{0}$ being a real constant, $E(t)=0$. Then, the particular solution of (28) is

$$
x_{p}(t)=A_{h} e^{-\frac{\gamma t}{2}} \cos \left(\Omega_{0} t-\theta_{h}\right)+A_{p} \cos \left(\omega t-\delta_{p}\right),
$$

where $A_{h}$ and $\theta_{h}$ are constants of the transient part such that $x_{p}(t)$ satisfies the initial conditions $x_{p}(0)$ $=0, \dot{x}_{p}(0)=0$, and the amplitude and phase shift of the steady-state part are

$$
A_{p}=\frac{D_{0}}{\sqrt{\left(\omega_{0}^{2}-\omega^{2}\right)^{2}+\gamma^{2} \omega^{2}}}, \quad \delta_{p}=\tan ^{-1}\left(\frac{\gamma \omega}{\omega_{0}^{2}-\omega^{2}}\right) .
$$

For given $\gamma$ and $\omega_{0}$, the driving frequency $\omega$ at which the amplitude $A_{p}(\omega)$ takes maximum is known as the resonance frequency. For this model, the resonance frequency $\omega=\omega_{\text {res }}$ and the corresponding maximum amplitude are

$$
\omega_{\text {res }}=\sqrt{\omega_{0}^{2}-\gamma^{2} / 2}, \quad A_{p}\left(\omega_{\text {res }}\right)=\frac{D_{0}}{\sqrt{\gamma^{2}\left(\omega_{0}^{2}-\frac{\gamma^{2}}{4}\right)}},
$$

provided that $\omega_{0}^{2}-\gamma^{2} / 2>0$.

In Fig. 3, we give a plot showing the time-evolution of the probability density $\rho_{0}(q, t)$, which corresponds to $k=0$ in (10), and $\alpha=0$ in (16). Precisely, Fig. 3(a) shows the evolution of the Gaussian wave packet when $x_{p}(t)=0$, and Fig. 3(b) shows how the center of the Gaussian wave packet oscillates according to the classical solution $x_{p}(t)=(4 / \sqrt{47}) \cos \left(\sqrt{23 / 2} t-\tan ^{-1} \sqrt{46}\right)$ at resonance frequency (transient parts are neglected in the plots). Similarly, in Fig. 4, we plot the probability density $\rho_{k}(q, t)$ for $k=2$, given by $(10)$, without displacement $x_{p}(t)=0$, and with the same periodic displacement $x_{p}(t)$ as in the previous figure, but since $k=2$ we observe also the trajectories of the two moving zeros.

\section{B. Caldirola-Kanai models with constant parameter $B(t)=B_{0}$}

Now, consider the generalized quantum Hamiltonian $\hat{H}_{g}(t)$, with $B(t)=B_{0}$ being any real constant. Then, for given $\gamma$ and $\omega_{0}$ in Eq. (23), Eq. (24) gives $-\left(B_{0}^{2}+\gamma B_{0}\right) \equiv \Lambda_{0}^{2} \leq \gamma^{2} / 4$ so that the values of $\Lambda_{0}^{2}$ are restricted as $-\omega_{0}^{2}<\Lambda_{0}^{2} \leq \gamma^{2} / 4$. The case $\Lambda_{0}^{2}>\gamma^{2} / 4$ corresponds to imaginary $B_{0}$, which is not allowed for a self-adjoint Hamiltonian. According to this, for the modified classical oscillator (25) with $\Omega_{d}^{2}$ given by (26), one has the following cases:

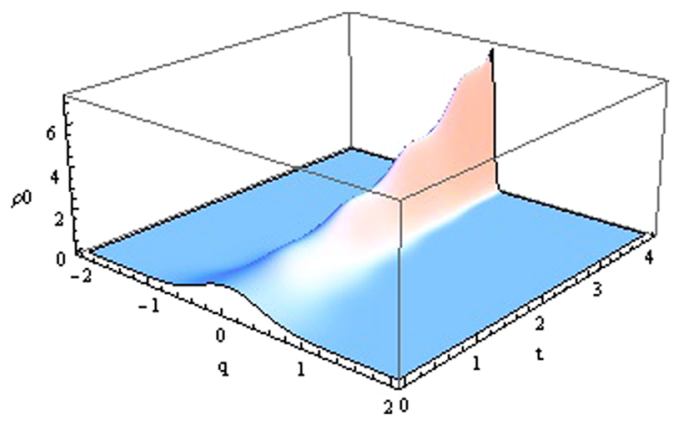

(a)

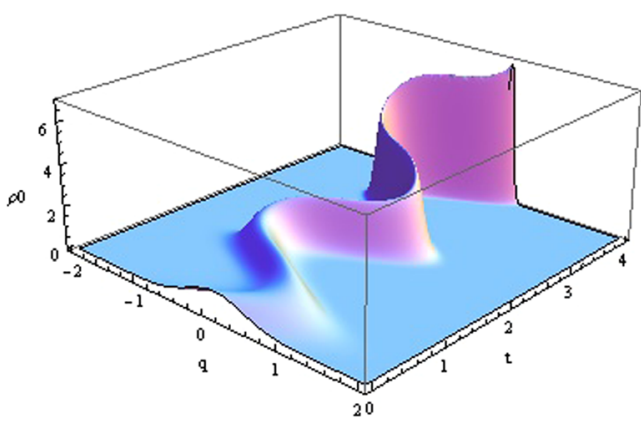

(b)

FIG. 3. Standard CK-model: probability density $\rho_{0}(q, t), \gamma=1$ and $\omega_{0}=\sqrt{12}, \hbar=1$, (a) with $x_{p}(t)=0$. (b) With periodic $x_{p}(t)$ at resonance frequency $\omega_{\text {res }}=\sqrt{23 / 2}, D_{0}=2$. 


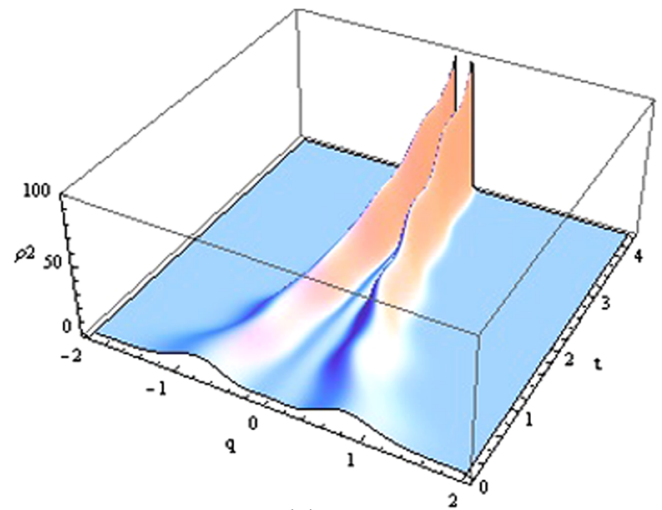

(a)

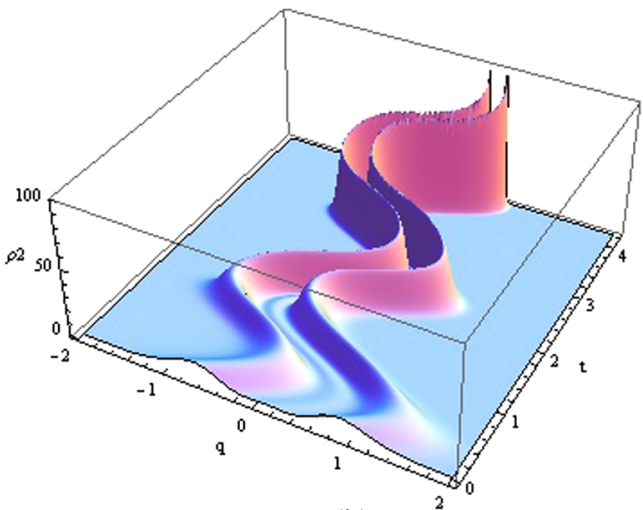

(b)

FIG. 4. Standard CK-model: probability density $\rho_{k}(q, t), k=2, \gamma=1$ and $\omega_{0}=\sqrt{12}, \hbar=1$, (a) with $x_{p}(t)=0$. (b) With periodic $x_{p}(t)$ at resonance frequency $\omega_{\text {res }}=\sqrt{23 / 2}, D_{0}=2$.

(i) $\Omega_{d}^{2}<0$, if $-\omega_{0}^{2}<\Lambda_{0}^{2}<-\omega_{0}^{2}+\gamma^{2} / 4$ (overdamping);

(ii) $\Omega_{d}^{2}=0$, if $\Lambda_{0}^{2}=-\omega_{0}^{2}+\gamma^{2} / 4$ (critical damping);

(iii) $\Omega_{d}^{2}>0$, if $-\omega_{0}^{2}+\gamma^{2} / 4<\Lambda_{0}^{2} \leq \gamma^{2} / 4$ (underdamping).

The corresponding frequency modification in momentum space is also constant $\Lambda_{p}^{2}=-B_{0}^{2}-\gamma B_{0}$, and the momentum equation becomes $\ddot{p}-\gamma \dot{p}+\left(\omega_{0}^{2}+\Lambda_{0}^{2}\right) p=0$. We note that different values of $B_{0}$ can give the same $\Lambda_{0}^{2}$. For example, both $B_{0}=0$ and $B_{0}=-\gamma$ give $\Lambda_{0}^{2}=0$ so that classical equations are the same, but the difference in $B_{0}$ will be reflected in the initial conditions of these equations.

For the case when the damping is weak, we shortly give the next basic estimates.

Underdamping case $\left(B(t)=B_{0}\right)$ : When $\Omega_{d}^{2}>0$, homogeneous solutions are

$$
x_{1}(t)=x_{0} \frac{\omega_{0}}{\Omega_{d}} e^{-\gamma t / 2} \cos \left(\Omega_{d} t-\delta\right), \quad x_{2}(t)=\frac{1}{x_{0} \Omega_{d}} e^{-\gamma t / 2} \sin \left(\Omega_{d} t\right),
$$

where $\Omega_{d}=\sqrt{\omega_{0}^{2}+\Lambda_{0}^{2}-\frac{\gamma^{2}}{4}}$ and $\delta=\tan ^{-1}\left[\left(\frac{\gamma}{2}+B_{0}\right) / \Omega_{d}\right]$. Therefore, the squeezing coefficient becomes

$$
R_{B}(t)=\frac{e^{\gamma t / 2}}{\sqrt{\left(\omega_{0}^{2} / \Omega_{d}^{2}\right)\left[\cos ^{2}\left(\Omega_{d} t-\delta\right)+\sin ^{2}\left(\Omega_{d} t\right)\right]}}
$$

and the uncertainty product is

$$
(\Delta \hat{q} \Delta \hat{p})_{\alpha}=\frac{\hbar}{2} \sqrt{1+\frac{\omega_{0}^{2}}{\Omega_{d}^{4}}\left[\left(\frac{\gamma}{2}+B_{0}\right)\left[\cos ^{2}\left(\Omega_{d} t-\delta\right)+\sin ^{2}\left(\Omega_{d} t\right)\right]+\frac{\Omega_{d}}{2}\left[\sin \left(2\left(\Omega_{d} t-\delta\right)\right)-\sin \left(2 \Omega_{d} t\right)\right]\right]^{2}} .
$$

Resonance $\left(B(t)=B_{0}\right)$ : Let $D(t)=D_{0} \cos (\omega t), E(t)=0$. Then, the particular solution is

$$
x_{p}(t)=A_{h} e^{-\frac{\gamma t}{2}} \cos \left(\Omega_{d} t-\theta_{h}\right)+A_{p} \cos \left(\omega t-\delta_{p}\right),
$$

where $A_{h}$ and $\theta_{h}$ are constants such that $x_{p}(t)$ satisfies the initial conditions $x_{p}(0)=0, \dot{x}_{p}(0)=0$, and the amplitude and phase shift of the steady-state part are

$$
A_{p}=\frac{D_{0}}{\sqrt{\left(\left(\omega_{0}^{2}+\Lambda_{0}^{2}\right)-\omega^{2}\right)^{2}+\gamma^{2} \omega^{2}}}, \quad \delta_{p}=\tan ^{-1}\left(\frac{\gamma \omega}{\left(\omega_{0}^{2}+\Lambda_{0}^{2}\right)-\omega^{2}}\right) .
$$


When $\omega=\sqrt{\omega_{0}^{2}+\Lambda_{0}^{2}}$ in (37), the amplitude as a function of $\gamma$ becomes $A_{p}(\gamma)=D_{0} / \sqrt{\gamma^{2}\left(\omega_{0}^{2}+\Lambda_{0}^{2}\right)}$. Thus, if $\gamma \rightarrow 0$, then the amplitude increases and tends to infinity for any $\omega_{0}^{2}$ and $\Lambda_{0}^{2}$, which is not surprising, since when $\gamma \rightarrow 0$, the behavior becomes close to the undamped motion for which resonance occurs at $\omega=\sqrt{\omega_{0}^{2}+\Lambda_{0}^{2}}$.

On the other side, for given $\gamma, \omega_{0}$, and $\Lambda_{0}^{2}$, the resonance frequency is $\omega_{\text {res }}=\sqrt{\left(\omega_{0}^{2}+\Lambda_{0}^{2}\right)-\gamma^{2} / 2}$, and the corresponding maximum amplitude and phase are

$$
A_{p}\left(\omega_{\text {res }}\right)=\frac{D_{0}}{\sqrt{\left(\omega_{0}^{2}+\Lambda_{0}^{2}\right) \gamma^{2}-\frac{\gamma^{4}}{4}}}, \quad \delta_{p}\left(\omega_{\text {res }}\right)=\tan ^{-1}\left(\frac{2 \omega_{\text {res }}}{\gamma}\right),
$$

provided that $\omega_{0}^{2}+\Lambda_{0}^{2}-\gamma^{2} / 2>0$. Therefore, for this model, resonance frequency exists, if for given $\gamma$ and $\omega_{0}$ the constant $\Lambda_{0}^{2}$ satisfies both $-\omega_{0}^{2}+\gamma^{2} / 4<\Lambda_{0}^{2} \leq \gamma^{2} / 4$ and $\omega_{0}^{2}+\Lambda_{0}^{2}-\gamma^{2} / 2>0$, that is, if $-\omega_{0}^{2}+\gamma^{2} / 2<\Lambda_{0}^{2} \leq \gamma^{2} / 4$. Such $\Lambda_{0}^{2}$ exists if and only if $\Omega_{0}^{2}=\omega_{0}^{2}-\gamma^{2} / 4>0$, that is, for the modified oscillator in the underdamping case resonance can occur only if the oscillator with $B(t)=0$ is also in the underdamping case. Now, when $\Lambda_{0}^{2}$ approaches the infimum value $-\omega_{0}^{2}+\gamma^{2} / 2$ from right, we have $\omega_{\text {res }} \rightarrow 0$ and $A_{p}\left(\omega_{\text {res }}\right) \rightarrow 2 D_{0} / \gamma^{2}$. The model for which $\Lambda_{0}^{2}$ takes its maximum value, that is, $\Lambda_{0}^{2}=\gamma^{2} / 4$, is interesting and will be discussed in Sec. IV, as the limiting case of Model 2.

Obviously, the qualitative behavior of the oscillator with a constant parameter $B(t)=B_{0}$ is similar to the standard CK-oscillator. However, the advantage could be that, for fixed $\gamma$ and $\omega_{0}$, by adding a suitable squeezing parameter $B_{0}$ to the system, one can change the behavior of the original oscillator $\left(B_{0}=0\right)$, from one case to another. For example, suppose $\Omega_{0}^{2}=\omega_{0}^{2}-\gamma^{2} / 4=0$ so that the original oscillator is in the critical case. Then, by adding a non-zero real constant $B_{0}$ to the model, one has $\Omega_{d}^{2}=\Lambda_{0}^{2}$, the sign of which will determine the case of the new oscillator.

\section{CALDIROLA-KANAI MODELS WITH TIME-VARIABLE PARAMETERS $B(t)$}

In this section, we introduce generalized Caldirola-Kanai oscillators with time-variable parameters $B(t)$. For this, we use that depending on the sign of $\Omega_{B}^{2}=\Lambda_{0}^{2}-\gamma^{2} / 4$, Eq. (24) also has different types of non-constant solutions such as

(i) $B(t)=-(\gamma / 2)+\Omega_{B}^{\prime} \tanh \left(\Omega_{B}^{\prime} t-\beta\right), \Omega_{B}^{\prime}=\sqrt{\left(\gamma^{2} / 4\right)-\Lambda_{0}^{2}}$, when $-\omega_{0}^{2}<\Lambda_{0}^{2}<\gamma^{2} / 4$,

(ii) $B(t)=-(\gamma / 2)+b /(1+b t)$, when $\Lambda_{0}^{2}=\gamma^{2} / 4$,

(iii) $B(t)=-(\gamma / 2)-\Omega_{B} \tan \left(\Omega_{B} t-\beta\right), \Omega_{B}=\sqrt{\Lambda_{0}^{2}-\left(\gamma^{2} / 4\right)}$ when $\Lambda_{0}^{2}>\gamma^{2} / 4$,

and their plot is given in Fig. 5. Here, $b$ is an arbitrary real constant and $\beta$ is an arbitrary phase shift, which we will take to be zero in order to avoid too many parameters. For these choices of $B(t)$, frequency modification $\Lambda_{0}^{2}$ in position space is again a constant by construction, but in momentum

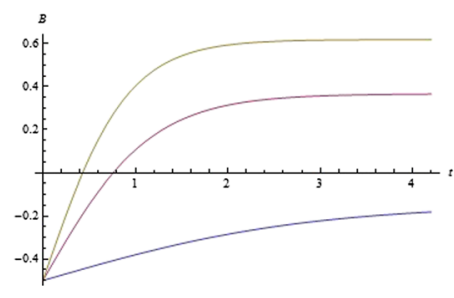

(a)

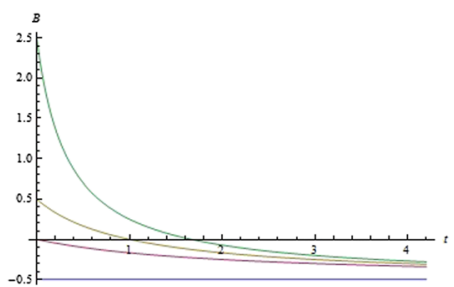

(b)

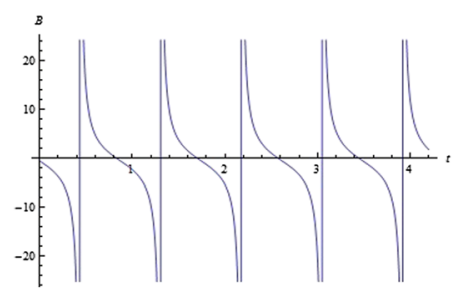

(c)

FIG. 5. (a) Model 1: $B(t)=-(\gamma / 2)+\Omega_{B}^{\prime} \tanh \left(\Omega_{B}^{\prime} t-\beta\right)$, with $\gamma=1, \Lambda_{0}^{2}=1 / 8,-1 / 2,-1, \beta=0$. (b) Model 2: $B(t)=-(\gamma / 2)+$ $b /(1+b t)$, with $\gamma=1$ and $b=0,1 / 2,1,3$. (c) Model 3: $B(t)=-(\gamma / 2)-\Omega_{B} \tan \left(\Omega_{B} t-\beta\right)$, with $\gamma=1, \Lambda_{0}^{2}=53 / 4, \beta=0$. 


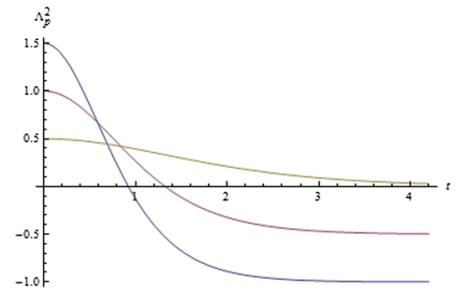

(a)

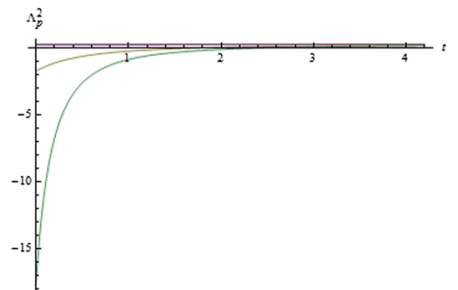

(b)

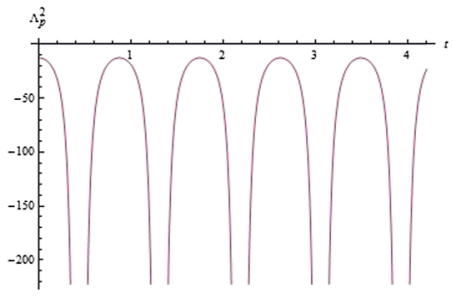

(c)

FIG. 6. Frequency modification $\Lambda_{p}^{2}(t)$ in momentum space. (a) Model 1 , with $\gamma=1, \Lambda_{0}^{2}=1 / 8,-1 / 2,-1$. (b) Model 2, with $\gamma=1$ and $b=0,1 / 2,1,3$. (c) Model 3, with $\gamma=1, \Lambda_{0}^{2}=53 / 4$.

space frequency modification $\Lambda_{p}^{2}(t)$ becomes time-dependent, as shown in Fig. 6. According to this, we study the following three models.

\section{A. Model 1}

First, we consider a generalized Caldirola-Kanai type quantum Hamiltonian $\hat{H}_{g}(t)$ given by (4), and $B(t)=-(\gamma / 2)+\Omega_{B}^{\prime} \tanh \left(\Omega_{B}^{\prime} t\right)$, where $\Omega_{B}^{\prime}=\sqrt{\left(\gamma^{2} / 4\right)-\Lambda_{0}^{2}}$ and $-\omega_{0}^{2}<\Lambda_{0}^{2}<\gamma^{2} / 4$. We note that the range of allowed $\Lambda_{0}^{2}$ here is the same as in the previous case for any real constant $B_{0}$. Also, $B(t)$ is minimum at $t=0$ with $B(0)=-\gamma / 2$, and as $t \rightarrow \infty$, it increases and asymptotically approaches the upper bound $B_{0}=-\frac{\gamma}{2}+\sqrt{\frac{\gamma^{2}}{4}-\Lambda_{0}^{2}}$; see Fig. 5(a). Then, we have

$$
\ddot{x}+\gamma \dot{x}+\left(\omega_{0}^{2}+\Lambda_{0}^{2}\right) x=D+e^{-\gamma t}(\dot{E}+B(t) E),
$$

with $\Omega_{d}^{2}=\omega_{0}^{2}+\Lambda_{0}^{2}-\gamma^{2} / 4$ and different possibilities, (i) $\Omega_{d}^{2}<0$ (ii) $\Omega_{d}^{2}=0$, and (iii) $\Omega_{d}^{2}>0$. The equation in momentum space becomes

$$
\ddot{p}-\gamma \dot{p}+\left(\omega_{0}^{2}+\Lambda_{p}^{2}(t)\right) p=e^{\gamma t}(\dot{D}-B(t) D)-\omega_{0}^{2} E,
$$

with time-dependent frequency modification

$$
\Lambda_{p}^{2}(t)=\frac{\gamma^{2}}{4}+\Omega_{B}^{\prime 2}\left(1-2 \tanh ^{2}\left(\Omega_{B}^{\prime} t\right)\right)
$$

which at $t=0$ is $\Lambda_{p}^{2}(0)=\frac{\gamma^{2}}{4}+\Omega_{B}^{\prime 2}$, and as $t \rightarrow \infty, \Lambda_{p}^{2}(t)$ decreases and approaches the frequency modification in position space $\Lambda_{0}^{2}=\left(\gamma^{2} / 4\right)-\Omega_{B}^{\prime 2}$; see Fig. 6(a).

Underdamping case (Model 1): If $\Omega_{d}^{2}>0$, that is, when $-\omega_{0}^{2}+\gamma^{2} / 4<\Lambda_{0}^{2}<\gamma^{2} / 4$, homogeneous solutions are

$$
x_{1}(t)=x_{0} e^{-\frac{\gamma t}{2}} \cos \left(\Omega_{d} t\right), \quad x_{2}(t)=\frac{1}{x_{0} \Omega_{d}} e^{-\frac{\gamma t}{2}} \sin \left(\Omega_{d} t\right),
$$

where $\Omega_{d}=\sqrt{\omega_{0}^{2}+\Lambda_{0}^{2}-\gamma^{2} / 4}$. Also, since $B(t)$ is smooth, solutions in momentum space

$$
\begin{aligned}
& p_{1}(t)=-x_{0} \Omega_{d} e^{\gamma t / 2}\left(\sin \left(\Omega_{d} t\right)+\left(\Omega_{B}^{\prime} / \Omega_{d}\right) \cos \left(\Omega_{d} t\right) \tanh \left(\Omega_{B}^{\prime} t\right)\right), \\
& p_{2}(t)=\frac{1}{x_{0}} e^{\gamma t / 2}\left(\cos \left(\Omega_{d} t\right)-\left(\Omega_{B}^{\prime} / \Omega_{d}\right) \sin \left(\Omega_{d} t\right) \tanh \left(\Omega_{B}^{\prime} t\right)\right)
\end{aligned}
$$

are also smooth. For this model, the squeezing coefficient is

$$
R_{B}(t)=\frac{e^{\gamma t / 2}}{\sqrt{\cos ^{2}\left(\Omega_{d} t\right)+\left(\omega_{0}^{2} / \Omega_{d}^{2}\right) \sin ^{2}\left(\Omega_{d} t\right)}}, \quad \frac{\omega_{0}^{2}}{\Omega_{d}^{2}}>1 .
$$


Since $\left(\omega_{0}^{2} / \Omega_{d}^{2}\right)>1, R_{B}(t)$ oscillates below the limiting squeezing $R_{0}(t)=e^{\gamma t / 2}$, and when $\Lambda_{0}^{2} \rightarrow \gamma^{2} / 4$, it approaches $R_{0}(t)=e^{\gamma t / 2}$. In other words, for given $\gamma$ and $\omega_{0}$, when the frequency $\Omega_{d}$ increases, the amplitude of oscillations of $R_{B}(t)$ decreases, as shown in Fig. 7(a).

Then, knowing $R_{B}(t)$, uncertainties $\Delta \hat{q}$ and $\Delta \hat{p}$ are of the form (20), (21), and plots showing explicitly their squeezing and spreading properties are given in Fig. 8. It follows that the corresponding uncertainty product is

$$
(\Delta \hat{q})_{\alpha}(\Delta \hat{p})_{\alpha}=\frac{\hbar}{2} \sqrt{1+\frac{1}{\omega_{0}^{2}}\left[\Omega_{B}^{\prime} \tanh \left(\Omega_{B}^{\prime} t\right)\left(\cos ^{2}\left(\Omega_{d} t\right)+\left(\frac{\omega_{0}^{2}}{\Omega_{d}^{2}}\right) \sin ^{2}\left(\Omega_{d} t\right)\right)-\left(\frac{\Omega_{B}^{\prime 2}}{2 \Omega_{d}}\right) \sin \left(2 \Omega_{d} t\right)\right]^{2}},
$$

which becomes minimum when $\Lambda_{0}^{2} \rightarrow \gamma^{2} / 4$, Fig. 7(b).

Resonance (Model 1): If the oscillator is in the underdamping case, and we have periodic forces $D(t)=D_{0} \cos (\omega t), E(t)=0$, then $x_{p}(t)$ has the same form as Eq. (36), and the resonance frequency is given by (38). Moreover, parameters $\gamma, \omega_{0}$, and $\Lambda_{0}^{2}$ satisfy the same conditions as for the underdamping case for $B(t)=B_{0}$, so the displacement of the wave packets at resonance frequency is the same. In Fig. 9 , the probability density $\rho_{0}(q, t)$ is plotted for $x_{p}(t)=0$ and for periodic $x_{p}(t)$ at resonance frequency.

As a result, we can say that Model 1 is very similar to the model with $B(t)=B_{0}$. The difference due to the modification in momentum space is felt only at times close to the initial time $t=0$, and as $t \rightarrow \infty$, both models show essentially the same behavior.

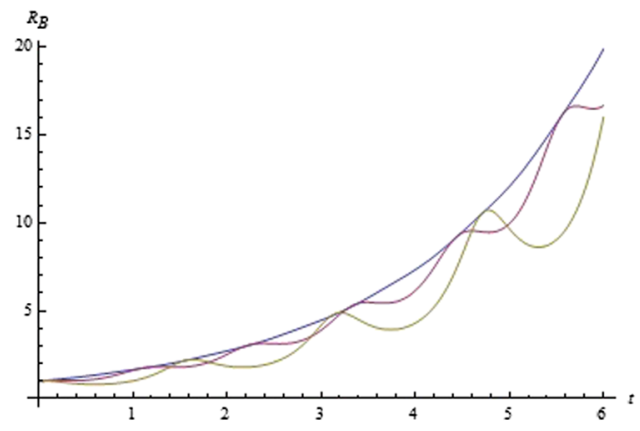

(a)

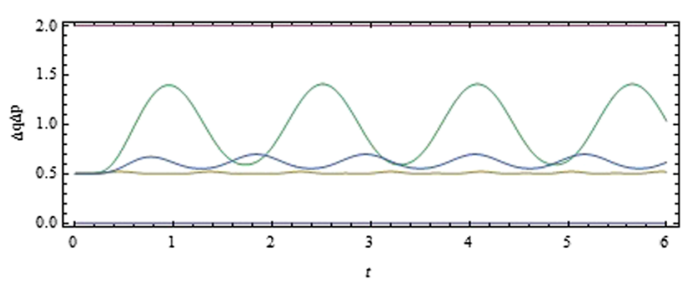

(b)

FIG. 7. Model 1: For $\omega_{0}=\sqrt{12}, \gamma=1, \quad \Lambda_{0}^{2}=0,-15 / 4,-31 / 4, \Omega_{d}=\sqrt{47} / 2, \sqrt{8} 2$. (a) $R_{B}(t)$. (b) Uncertainty product $(\Delta \hat{q})_{\alpha}(\Delta \hat{p})_{\alpha}(t)$.

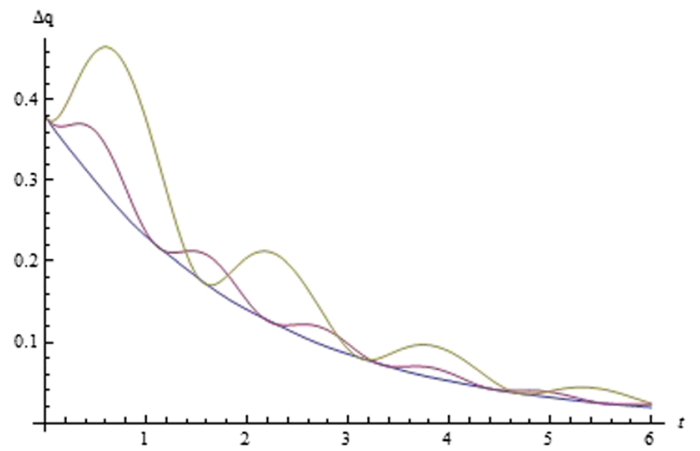

(a)

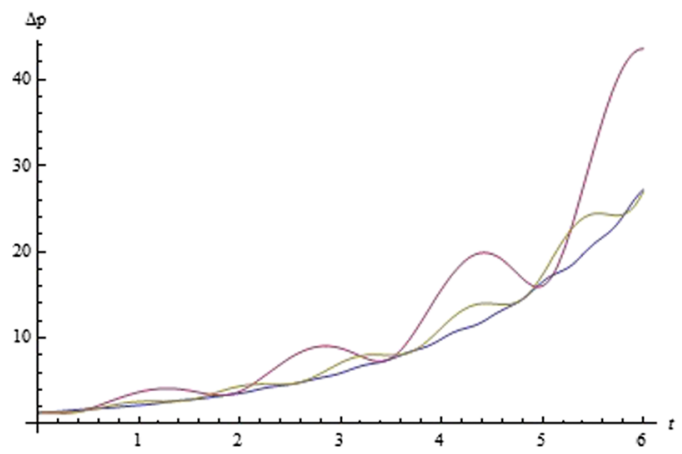

(b)

FIG. 8. Model 1: For $\omega_{0}=\sqrt{12}, \gamma=1, \Lambda_{0}^{2}=0,-15 / 4,-31 / 4 . \Omega_{d}=\sqrt{47} / 2, \sqrt{8}, 2, \Omega_{B}^{\prime}=1 / 2,2, \sqrt{8}$. (a) Uncertainty $\Delta \hat{q}$. (b) Uncertainty $\Delta \hat{p}$. 


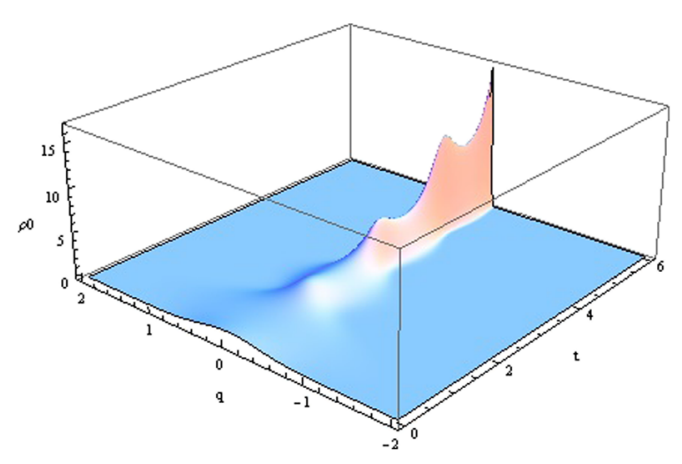

(a)

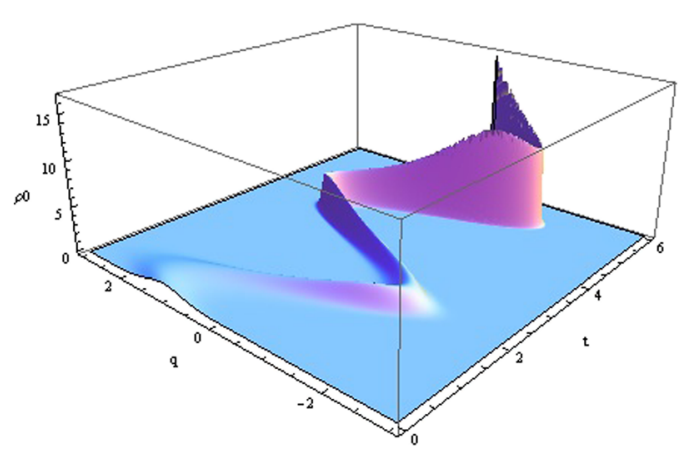

(b)

FIG. 9. Model 1: Probability density $\rho_{0}(q, t)$ with $\gamma=1, \omega_{0}=\sqrt{12}, \Lambda_{0}^{2}=-31 / 4, \hbar=1$ (a) $\rho_{0}(q, t)$ without displacement and (b) $\rho_{0}(q, t)$ displaced by periodic $x_{p}(t)$ and resonance frequency $\omega=\sqrt{15} / 2, D_{0}=3$.

\section{B. Model 2}

Next, we consider the general Hamiltonian $\hat{H}_{g}(t)$ given by (4) with $B(t)=-(\gamma / 2)+b /(1+b t)$, where $b$ is an arbitrary constant. If $b=0$, then $B(t)=-\gamma / 2$ is constant. If $b<0$, then $B(t)$ has a finite time singularity at $t=-1 / b$. Otherwise, for $b>0, B(t)$ is maximum at $t=0$ and smoothly decreases with increasing time, asymptotically approaching the lower bound $B(t)=-\gamma / 2$, Fig. 5(b). Since $-\left(\dot{B}+B^{2}+\gamma B\right)=\gamma^{2} / 4$ for any real constant $b$, then $\Lambda_{0}^{2}=\gamma^{2} / 4$ and the frequency of the modified damped oscillator $\Omega_{d}=\sqrt{\omega_{0}^{2}+\Lambda_{0}^{2}-\frac{\gamma^{2}}{4}}$ becomes equal to the frequency of the undamped one, that is, $\Omega_{d}=\omega_{0}$. Thus, no matter what is the sign of $\Omega_{0}^{2}=\omega_{0}^{2}-\gamma^{2} / 4$ for the original oscillator, if one adds to the system $B(t)$ defined here, the new oscillator always becomes in the special underdamping case, with $\Omega_{d}=\omega_{0}$. The corresponding classical equation of motion is

$$
\ddot{x}+\gamma \dot{x}+\left(\omega_{0}^{2}+\frac{\gamma^{2}}{4}\right) x=D+e^{-\gamma t}(\dot{E}+B(t) E),
$$

with homogeneous solutions

$$
x_{1}(t)=\frac{x_{0}}{\omega_{0}} \sqrt{\left(\omega_{0}^{2}+b^{2}\right)} e^{-\frac{\gamma t}{2}} \cos \left(\omega_{0} t-\delta\right), \quad x_{2}(t)=\frac{1}{\omega_{0} x_{0}} e^{-\frac{\gamma t}{2}} \sin \left(\omega_{0} t\right),
$$

where $\delta=\tan ^{-1}\left(b / \omega_{0}\right)$, and $x_{1}(0)=x_{0} \neq 0, \dot{x}_{1}(0)=(-\gamma / 2+b) x_{0} ; x_{2}(0)=0, \dot{x}_{2}(0)=1 / x_{0}$. Note that the constant $b$ enters the homogeneous solution via the necessary condition $\dot{x}_{1}(0)=x_{0} B(0)$ but does not appear explicitly in Eq. (45). The equation in momentum space becomes

$$
\ddot{p}-\gamma \dot{p}+\left(\omega_{0}^{2}+\Lambda_{p}^{2}(t)\right) p=e^{\gamma t}(\dot{D}-B(t) D)-\omega_{0}^{2} E,
$$

with frequency modification depending on time

$$
\Lambda_{p}^{2}(t)=\frac{\gamma^{2}}{4}-\frac{2 b^{2}}{(1+b t)^{2}}
$$

which is smooth for $b \geq 0$ and has singularity at $t=-1 / b$ for negative $b$. Also, as $t \rightarrow \infty$, it approaches the constant modification in position space, that is, $\Lambda_{p}^{2}(t) \rightarrow \gamma^{2} / 4$, Fig. 6(b). Homogeneous solutions of (47) are

$$
\begin{array}{r}
p_{1}(t)=-\frac{x_{0}}{\omega_{0}} \sqrt{\left(\omega_{0}^{2}+b^{2}\right)} e^{\gamma t / 2}\left(\omega_{0} \sin \left(\omega_{0} t-\delta\right)+\frac{b}{1+b t} \cos \left(\omega_{0} t-\delta\right)\right), \\
p_{2}(t)=\frac{1}{\omega_{0} x_{0}} e^{\gamma t / 2}\left(\omega_{0} \cos \left(\omega_{0} t\right)-\frac{b}{1+b t} \sin \left(\omega_{0} t\right)\right),
\end{array}
$$

which could have singularity only for negative $b$. 
In Model 2, the squeezing coefficient takes the form

$$
R_{B}(t)=\frac{e^{\frac{\gamma t}{2}}}{\sqrt{\left(\frac{\omega_{0}^{2}+b^{2}}{\omega_{0}^{2}}\right) \cos ^{2}\left(\omega_{0} t-\delta\right)+\sin ^{2}\left(\omega_{0} t\right)}},
$$

and since it is smooth for any constant $b$, probability densities are also smooth. For the limiting case $b=0$, one has $R_{B}(t)=e^{\frac{\gamma t}{2}}$, leading to monotone squeezing of the wave packets. Otherwise, for $b \neq 0$, the coefficient $R_{B}(t)$ has oscillatory behavior. Moreover, for this model, we have fixed frequency $\omega_{0}$, but the amplitude of the oscillations of $R_{B}(t)$ can be increased arbitrarily by increasing the value of $|b|$; see Fig. 10(a). Thus, knowing $R_{B}(t)$, uncertainties $\Delta \hat{q}$ and $\Delta \hat{p}$ are of the form (20), (21), and their behavior for $b=0,1,3,6$ is shown in Fig. 11. In Fig. 12, one can see the phase-plane diagram. The uncertainty relation is

$$
(\Delta \hat{q})_{\alpha}(\Delta \hat{p})_{\alpha}(t)=
$$

$$
\frac{\hbar}{2} \sqrt{1+\frac{1}{4}\left[\frac{\left(2 b / \omega_{0}\right)}{(1+b t)}\left(\frac{\omega_{0}^{2}+b^{2}}{\omega_{0}^{2}} \cos ^{2}\left(\omega_{0} t-\delta\right)+\sin ^{2}\left(\omega_{0} t\right)\right)+\frac{\omega_{0}^{2}+b^{2}}{\omega_{0}^{2}} \sin \left(2\left(\omega_{0} t-\delta\right)\right)-\sin \left(2 \omega_{0} t\right)\right]^{2}},
$$

which is oscillatory for $b \neq 0$, and for $b=0$ one has $(\Delta \hat{q})_{\alpha}(\Delta \hat{p})_{\alpha}=\hbar / 2$, Fig. 10(b). Clearly, if $B(t)$ has singularity, it is reflected in momentum and the uncertainty product as well.

Resonance (Model 2). Let $D(t)=D_{0} \cos (\omega t)$, and $E(t)=0$ in (45). Then,

$$
\begin{array}{r}
x_{p}(t)=x_{h}(t)+A_{p} \cos \left(\omega t-\delta_{p}\right), \\
p_{p}(t)=e^{\gamma t}\left(\dot{x}_{p}(t)-\left(-\frac{\gamma}{2}+\frac{b}{1+b t}\right) x_{p}(t)\right),
\end{array}
$$

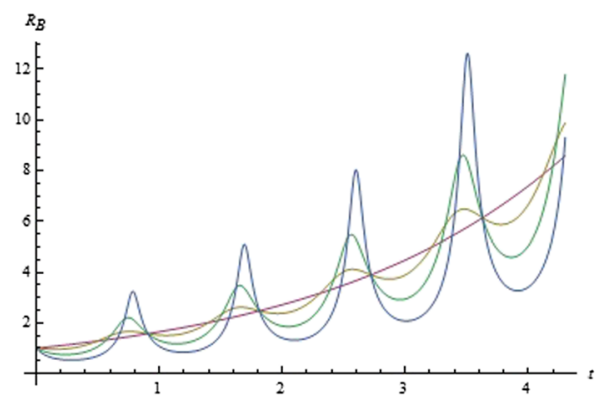

(a)

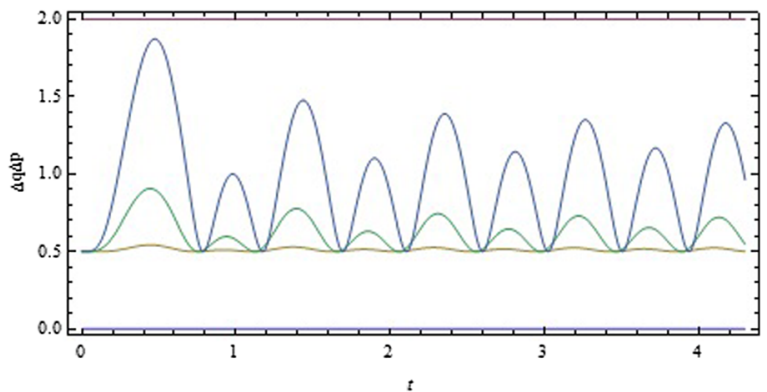

(b)

FIG. 10. Model 2: $\omega_{0}=\sqrt{12}, \gamma=1$, and $b=0,1,3,6, \hbar=1$. (a) $R_{B}(t)$; (b) uncertainty product $(\Delta \hat{q})_{\alpha}(\Delta \hat{p})_{\alpha}$.

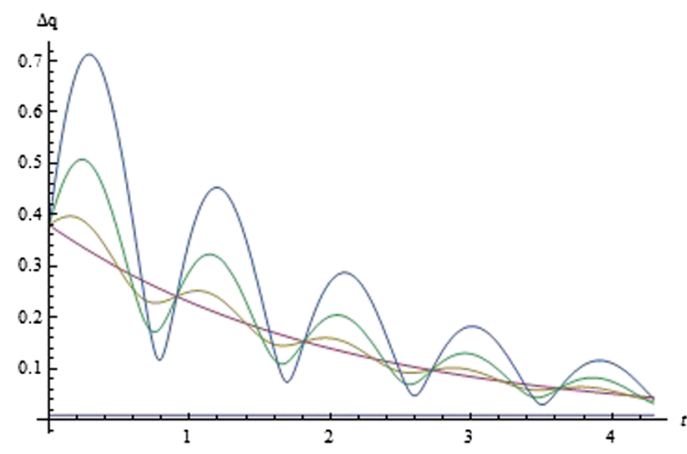

(a)

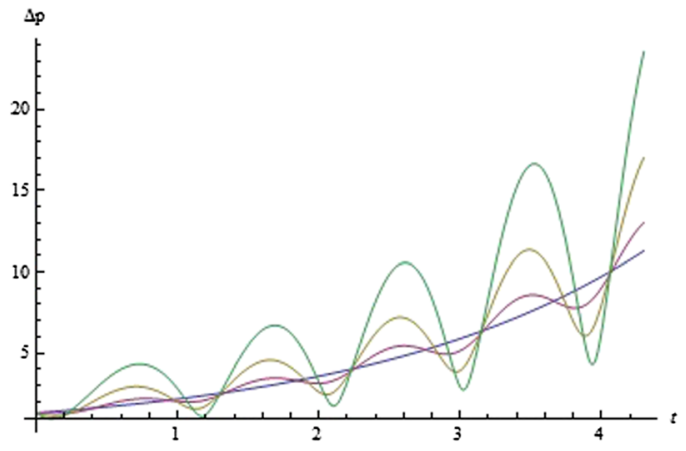

(b)

FIG. 11. Model 2: $\omega_{0}=\sqrt{12}, \gamma=1$, and $b=0,1,3,6, \hbar=1$. (a) Uncertainty $\Delta \hat{q}(t)$. (b) Uncertainty $\Delta \hat{p}(t)$. 


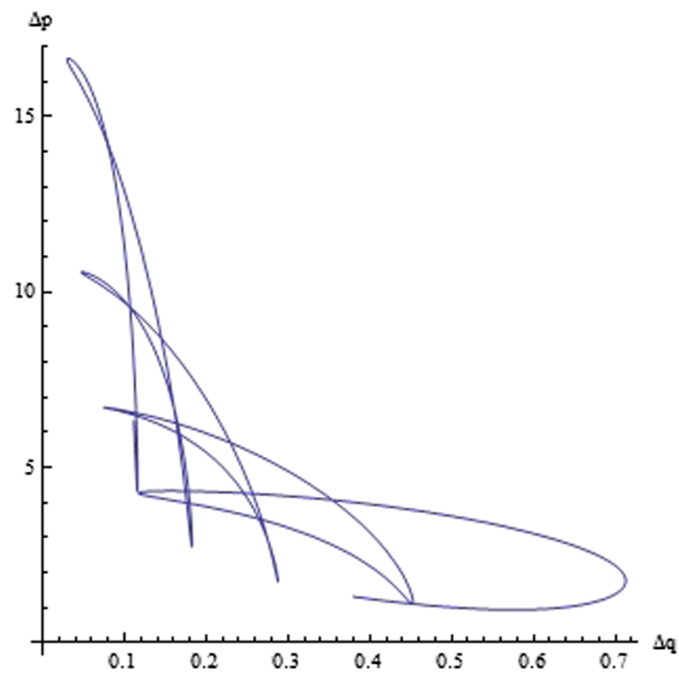

(a)

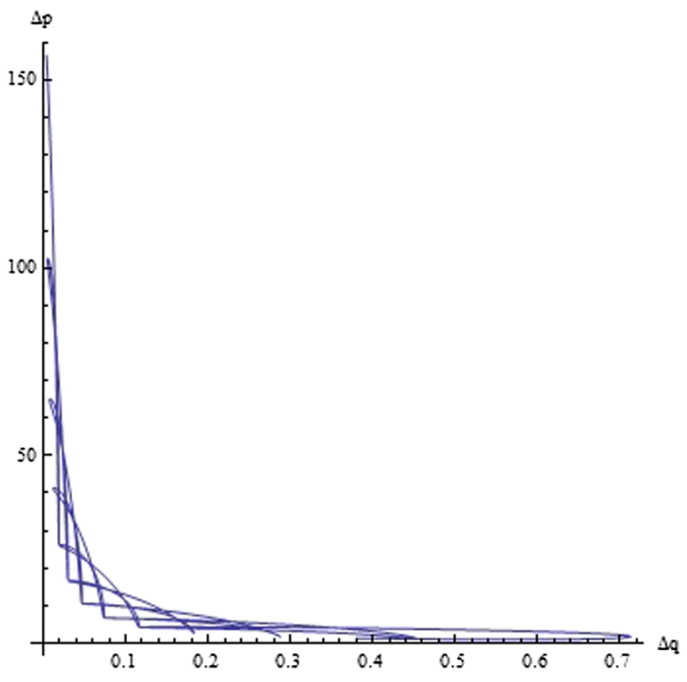

(b)

FIG. 12. Model 2: $\omega_{0}=\sqrt{12}, \gamma=1$, and $b=6, \hbar=1$. $(\Delta \hat{q}(t), \Delta \hat{p}(t))$. (a) $0 \leq t \leq 4$. (b) $0 \leq t \leq 8$.

where $x_{h}(t)$ is the transient part such that $x_{p}(t)$ satisfies initial conditions $x_{p}(0)=0, \dot{x}_{p}(0)=0$. The amplitude and phase shift of the steady-state part of $x_{p}(t)$ take the form

$$
A_{p}=\frac{D_{0}}{\sqrt{\left(\left(\omega_{0}^{2}+\frac{\gamma^{2}}{4}\right)-\omega^{2}\right)^{2}+\gamma^{2} \omega^{2}}}, \quad \delta_{p}=\tan ^{-1}\left(\frac{\gamma \omega}{\left(\omega_{0}^{2}+\frac{\gamma^{2}}{4}\right)-\omega^{2}}\right)
$$

so that resonance frequency and maximum amplitude for $x_{p}(t)$ become

$$
\omega_{\text {res }}=\sqrt{\omega_{0}^{2}-\frac{\gamma^{2}}{4}}=\Omega_{0}, \quad A_{p}\left(\omega_{\text {res }}\right)=\frac{D_{0}}{\omega_{0} \gamma},
$$

provided that $\Omega_{0}^{2}=\omega_{0}^{2}-\frac{\gamma^{2}}{4}>0$. It means that resonance in the present modified oscillator can occur only if the original oscillator $(B(t)=0)$ is also in the underdamping case.

In Fig. 13, we show the probability density $\rho_{k}(q, t)$ for $k=2$, when $b=0$ (a) without displacement and (b) with displacement under a periodic force with resonance frequency. As expected, for $b=0$, we observe monotone changes in amplitude and squeezing. In Fig. 14, we plot the probability density

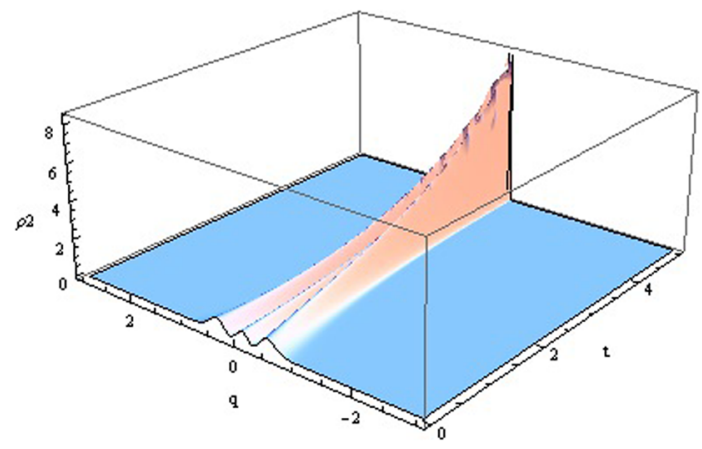

(a)

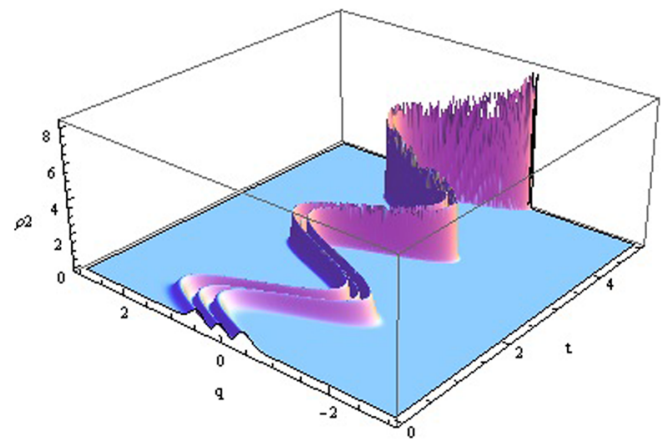

(b)

FIG. 13. Model 2: $\omega_{0}=\sqrt{12}, \gamma=1, b=0$. Probability density $\rho_{k}(q, t)$ for $k=2$. (a) With $x_{p}=0$. (b) With periodic $x_{p}(t)$, and resonance frequency $\omega=\sqrt{47} / 2, D_{0}=\sqrt{12}$. 


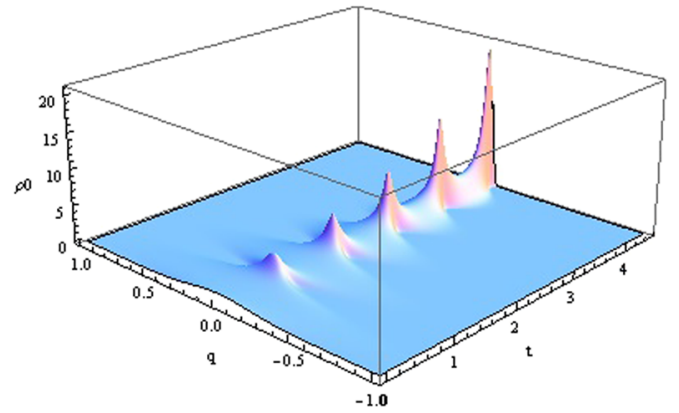

(a)

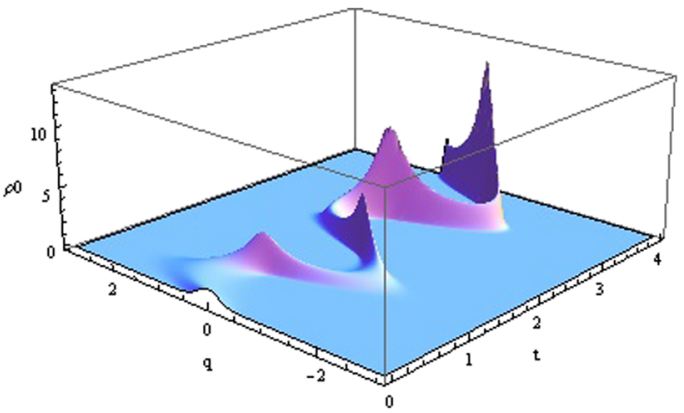

(b)

FIG. 14. Model 2: $\omega_{0}=\sqrt{12}, \gamma=1, b=6$. Probability density $\rho_{0}(q, t)$, (a) with $x_{p}(t)=0$ and (b) with periodic $x_{p}(t)$ at resonance frequency $\omega=\sqrt{47} / 2, D_{0}=\sqrt{12}$.

$\rho_{0}(q, t)$ when $b=6$, without displacement and with periodic displacement at resonance frequency, and observe the oscillatory squeezing in $\Delta \hat{q}$. Then, in Fig. 15, we depict the probability density $\rho_{k}(q, t)$ for $k=2$, when $b=6$.

\section{Model 3}

Now consider the quantum Hamiltonian $\hat{H}_{g}(t)$ given by (4) with $B(t)=-(\gamma / 2)-\Omega_{B} \tan \left(\Omega_{B} t\right)$, where $\Omega_{B}=\sqrt{\Lambda_{0}^{2}-\gamma^{2} / 4}$ and $\Lambda_{0}^{2}>\gamma^{2} / 4$. In this model, $B(t)$ is periodic, with singularities at $t=(n-1 / 2) \pi) / \Omega_{B}, n=1,2, \ldots$, and see Fig. $5(\mathrm{c})$. The corresponding classical equation is

$$
\ddot{x}+\gamma \dot{x}+\left(\omega_{0}^{2}+\Lambda_{0}^{2}\right) x=D+e^{-\gamma t}(\dot{E}+B E),
$$

where $\Omega_{d}^{2}=\omega_{0}^{2}+\Lambda_{0}^{2}-\gamma^{2} / 4$. Since $\Lambda_{0}^{2}-\gamma^{2} / 4>0$ implies $\Omega_{d}^{2}>0$ for any $\gamma$ and $\omega_{0}$, the original oscillator $(B=0)$ could be critically overdamped or underdamped, but the modified oscillator is always in the underdamping case. Then, homogeneous solutions are

$$
x_{1}(t)=x_{0} e^{-\frac{\gamma t}{2}} \cos \left(\Omega_{d} t\right), \quad x_{2}(t)=\frac{1}{x_{0} \Omega_{d}} e^{-\frac{\gamma t}{2}} \sin \left(\Omega_{d} t\right),
$$

where $\Omega_{d}=\sqrt{\omega_{0}^{2}+\Lambda_{0}^{2}-\gamma^{2} / 4}$, and they satisfy $x_{1}(0)=x_{0} \neq 0, \dot{x}_{1}(0)=-\gamma x_{0} / 2 ; x_{2}(0)=0$, $\dot{x}_{2}(0)=1 / x_{0}$. Here, the equation in momentum space is

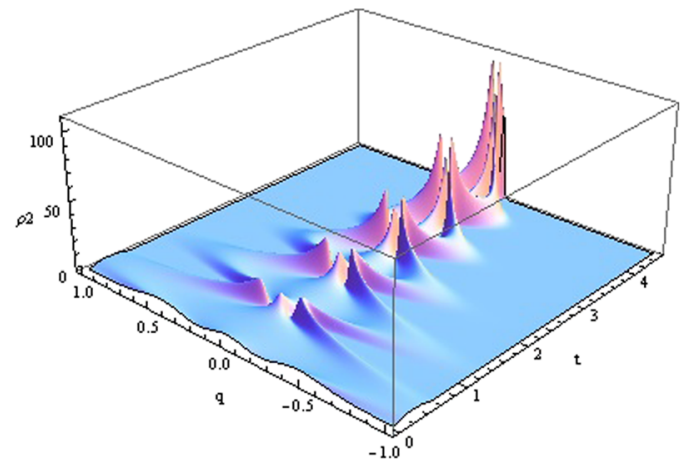

(a)

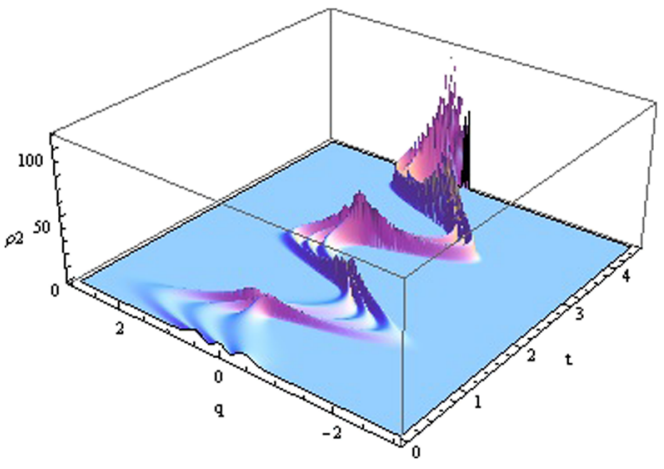

(b)

FIG. 15. Model 2: $\omega_{0}=\sqrt{12}, \gamma=1, b=6$. Probability density $\rho_{k}(q, t), k=2$. (a) With $x_{p}(t)=0$. (b) With periodic $x_{p}(t)$ at resonance frequency $\omega=\sqrt{47} / 2, D_{0}=\sqrt{12}$. 


$$
\ddot{p}-\gamma \dot{p}+\left(\omega_{0}^{2}+\Lambda_{p}^{2}(t)\right) p=e^{\gamma t}(\dot{D}-B D)-\omega_{0}^{2} E,
$$

where the modification of the frequency

$$
\Lambda_{p}^{2}(t)=\frac{\gamma^{2}}{4}-\Omega_{B}^{2}\left(1+2 \tan ^{2}\left(\Omega_{B} t\right)\right)
$$

is periodic and reflects the singularities of $B(t)$; see Fig. 6(c). It follows that solutions of (53),

$$
\begin{array}{r}
p_{1}(t)=x_{0} \Omega_{d} e^{\gamma t / 2}\left(-\sin \left(\Omega_{d} t\right)+\left(\Omega_{B} / \Omega_{d}\right) \cos \left(\Omega_{d} t\right) \tan \left(\Omega_{B} t\right)\right), \\
p_{2}(t)=\frac{1}{x_{0}} e^{\gamma t / 2}\left(\cos \left(\Omega_{d} t\right)+\left(\Omega_{B} / \Omega_{d}\right) \sin \left(\Omega_{d} t\right) \tan \left(\Omega_{B} t\right)\right),
\end{array}
$$

also have singularities at the same times as $B(t)$, which is expected. For this model, the squeezing coefficient becomes

$$
R_{B}(t)=\frac{e^{\gamma t / 2}}{\sqrt{\cos ^{2}\left(\Omega_{d} t\right)+\left(\omega_{0}^{2} / \Omega_{d}^{2}\right) \sin ^{2}\left(\Omega_{d} t\right)}}, \quad \frac{\omega_{0}^{2}}{\Omega_{d}^{2}}<1,
$$

which is smooth, and since $\left(\omega_{0}^{2} / \Omega_{d}^{2}\right)<1$, it oscillates above $R_{0}(t)=e^{\gamma t / 2}$. When $\left(\omega_{0}^{2} / \Omega_{d}^{2}\right) \rightarrow 1, R_{B}(t)$ approaches the limiting squeezing, that is, $R_{B}(t) \rightarrow e^{\gamma t / 2}$. Moreover, for given $\gamma$ and $\omega_{0}$, when $\Lambda_{0}$ increases, the frequency $\Omega_{d}$ and the amplitude of oscillations also increase. For example, taking $\gamma=1, \omega_{0}^{2}=12$, and then for the different values $\Lambda_{0}^{2}=1 / 4,53 / 4,209 / 4$, one gets, respectively, $\Omega_{d}=2 \sqrt{3}, 5,8$, and this behavior of $R_{B}(t)$ is shown in Fig. 16(a).

Using $R_{B}(t)$ given by (56), uncertainties are of the form

$$
\begin{gathered}
(\Delta \hat{q})_{\alpha}=\sqrt{\frac{\hbar}{2 \omega_{0}}} \frac{1}{R_{B}(t)}, \\
(\Delta \hat{p})_{\alpha}=\sqrt{\frac{\omega_{0} \hbar}{2}} R_{B}(t) \sqrt{1+\frac{e^{2 \gamma t}}{\left(\omega_{0} R_{B}^{2}(t)\right)^{2}}\left(\frac{\dot{R}_{B}(t)}{R_{B}(t)}+\left(-(\gamma / 2)-\Omega_{B} \tan \left(\Omega_{B} t\right)\right)\right)^{2}},
\end{gathered}
$$

and the uncertainty relation is explicitly found,

$$
(\Delta \hat{q})_{\alpha}(\Delta \hat{p})_{\alpha}(t)=\frac{\hbar}{2} \sqrt{1+\frac{1}{\omega_{0}^{2}}\left[\Omega_{B} \tan \left(\Omega_{B} t\right)\left(\cos ^{2}\left(\Omega_{d} t\right)+\left(\frac{\omega_{0}^{2}}{\Omega_{d}^{2}}\right) \sin ^{2}\left(\Omega_{d} t\right)\right)-\left(\frac{\Omega_{B}^{2}}{2 \Omega_{d}}\right) \sin \left(2 \Omega_{d} t\right)\right]^{2}} .
$$

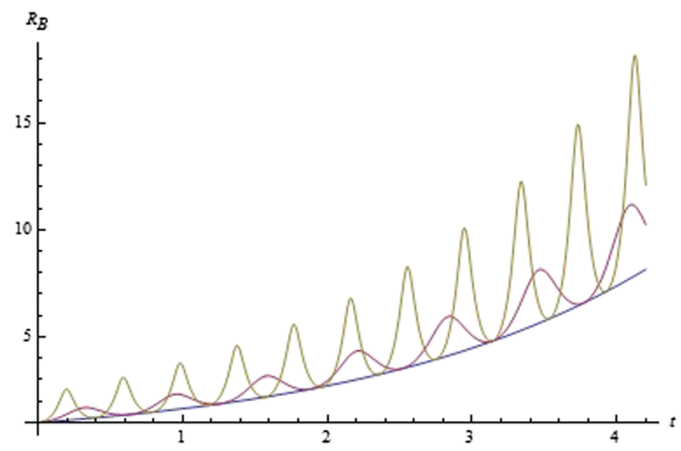

(a)

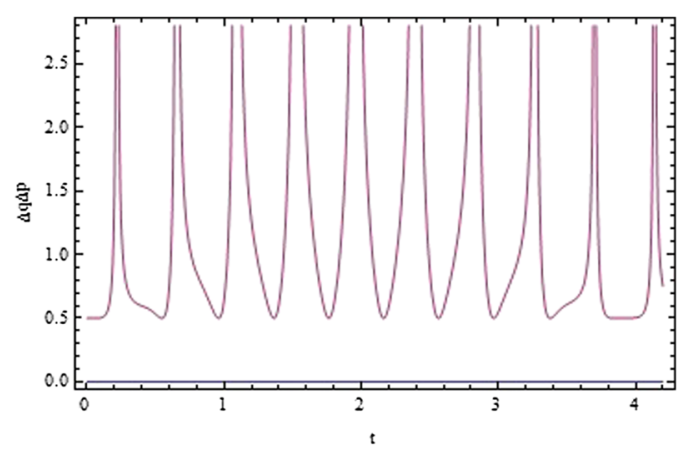

(b)

FIG. 16. Model 3: (a) $R_{B}(t)$ for $\omega_{0}=\sqrt{12}$, with $\gamma=1$, and $\Lambda_{0}=1 / 2, \sqrt{53} / 2, \sqrt{209} / 2$. (b) Uncertainty product $\Delta \hat{q} \Delta \hat{p}$ at coherent states, $\omega_{0}=\sqrt{12}, \gamma=1, \Lambda_{0}=\sqrt{209} / 2, \Omega_{d}=8, \hbar=1$. 
For this model, the uncertainty of position $(\Delta \hat{q})_{\alpha}$ is smooth and oscillatory, and approaches zero as time increases; however, the uncertainty of momentum $(\Delta \hat{p})_{\alpha}$ and the uncertainty product have singularities at points where $B(t)$ is singular; see Figs. 16(b) and 17.

Resonance (Model 3): Consider again the periodic forcing $D(t)=D_{0} \cos (\omega t)$, with $D_{0}$ being a real constant, $E(t)=0$. Then, a particular solution of (51) satisfying the initial conditions $x_{p}(0)=0$, $\dot{x}_{p}(0)=0$ is of the form

$$
x_{p}(t)=A_{h} e^{-\frac{\gamma t}{2}} \cos \left(\Omega_{d} t-\theta_{h}\right)+A_{p} \cos \left(\omega t-\delta_{p}\right),
$$

with the resonance frequency and amplitude given by

$$
\omega_{\text {res }}=\sqrt{\left(\omega_{0}^{2}+\Lambda_{0}^{2}\right)-\gamma^{2} / 2}, \quad A_{p}\left(\omega_{\text {res }}\right)=\frac{D_{0}}{\sqrt{\left(\omega_{0}^{2}+\Lambda_{0}^{2}\right) \gamma^{2}-\frac{\gamma^{4}}{4}}},
$$

where $\Lambda_{0}^{2}>\gamma^{2} / 4$. Thus, resonance in this modified oscillator will always occur if the original oscillator is in the underdamped or critical case, that is, if $\omega_{0}^{2}-\gamma^{2} / 4 \geq 0$ together with the condition $\Lambda_{0}^{2}-\gamma / 4>0$. However, for this model, it is possible to have resonance even when $\omega_{0}^{2}-\gamma^{2} / 4<0$. Moreover, for fixed $\gamma$ and $\omega_{0}$, resonance can be controlled by parameter $\Lambda_{0}^{2}$. For example, since $\Lambda_{0}^{2}>\gamma^{2} / 4$, the maximum amplitude will increase when $\Lambda_{0}^{2}$ decreases and approaches $\gamma^{2} / 4$, but it has an upper bound, that is, $A_{p}\left(\omega_{\text {res }}\right)<D_{0} / \omega_{0} \gamma$. From another side, by increasing the value of $\Lambda_{0}^{2}$, one can decrease the amplitude $A_{p}\left(\omega_{\text {res }}\right)$, at the expense of increasing the resonance frequency $\omega_{\text {res }}$.

Now, knowing $x_{p}(t)$, we have $p_{p}(t)=e^{\gamma t}\left(\dot{x}_{p}(t)+\left[\gamma / 2+\Omega_{B} \tan \left(\Omega_{B} t\right)\right] x_{p}(t)\right)$, and expectation values at coherent states become

$$
\begin{aligned}
\langle\hat{q}\rangle_{\alpha}(t)= & \sqrt{\frac{2 \hbar}{\omega_{0}}} e^{-\gamma t / 2}\left(\alpha_{1} \cos \left(\Omega_{d} t\right)+\alpha_{2}\left(\omega_{0} / \Omega_{d}\right) \sin \left(\Omega_{d} t\right)\right)+x_{p}(t), \\
\langle\hat{p}\rangle_{\alpha}(t) & =\sqrt{\frac{2 \hbar}{\omega_{0}}} e^{\gamma t / 2}\left(-\alpha_{1}\left(\Omega_{d} \sin \left(\Omega_{d} t\right)-\Omega_{B} \cos \left(\Omega_{d}\right) \tan \left(\Omega_{B} t\right)\right)\right. \\
& \left.+\alpha_{2} \omega_{0}\left(\cos \left(\Omega_{d} t\right)+\left(\Omega_{B} / \Omega_{d}\right) \sin \left(\Omega_{d} t\right) \tan \left(\Omega_{B} t\right)\right)\right)+p_{p}(t) .
\end{aligned}
$$

Therefore, singularities of the squeezing parameter $B(t)$ are reflected in momentum uncertainty, uncertainty product, and expectations of momentum, but the squeezing coefficient $R_{B}(t)$, probability densities, and expectations of position remain smooth. In Fig. 18(a), we plot the probability density $\rho_{0}(q, t)$, and observe oscillatory squeezing of the width, much more like "breathing" of the squeezing wave. Then, in Fig. 18(b), we show $\rho_{0}(q, t)$, under periodic displacement $x_{p}(t)$ at resonance frequency. Finally, in Fig. 19, we show the behavior of $\rho_{k}(q, t)$ for $k=2$.

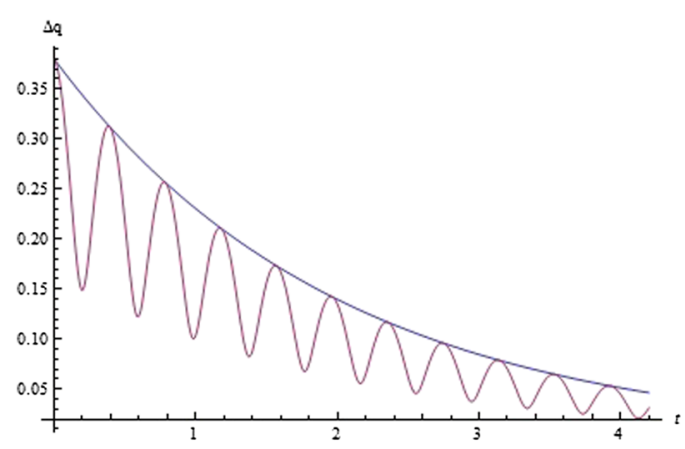

(a)

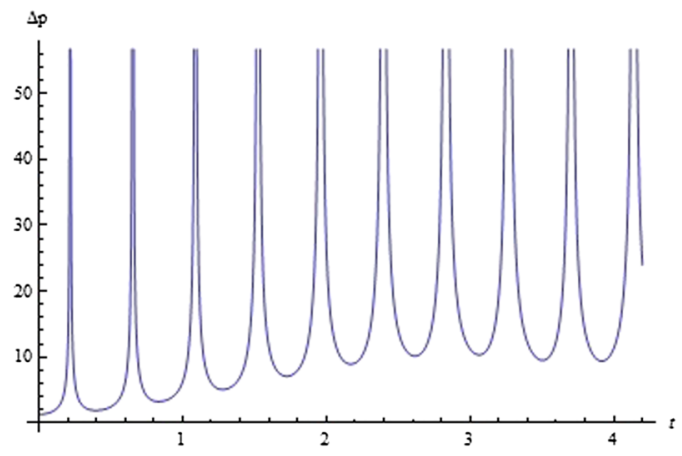

(b)

FIG. 17. Model 3: $\omega_{0}=\sqrt{12}, \gamma=1$, and $\Lambda_{0}=\sqrt{209} / 2, \Omega_{d}=8, \hbar=1$. (a) Uncertainty $\Delta \hat{q}$. (b) Uncertainty $\Delta \hat{p}$. 


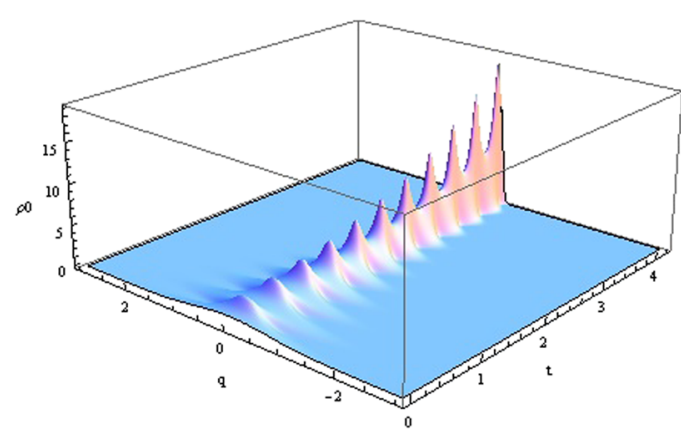

(a)

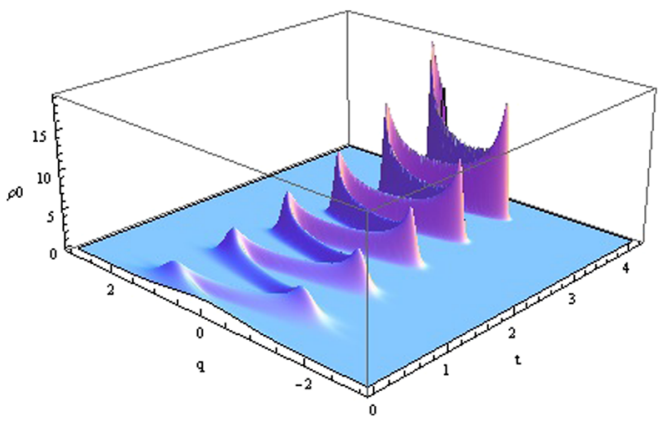

(b)

FIG. 18. Model 3: Probability density $\rho_{0}(q, t)$ with $\gamma=1, \omega_{0}=\sqrt{12}, \Lambda_{0}=\sqrt{209} / 2, \Omega_{d}=8, \hbar=1$, (a) $\rho_{0}(q, t)$ with $x_{p}(t)=0$ and (b) $\rho_{0}(q, t)$ displaced by periodic $x_{p}(t)$ at resonance frequency $\omega=\sqrt{255} / 2, D_{0}=8$.

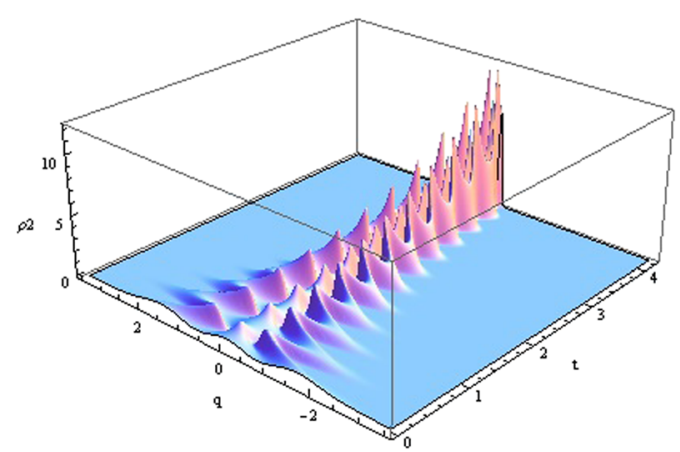

(a)

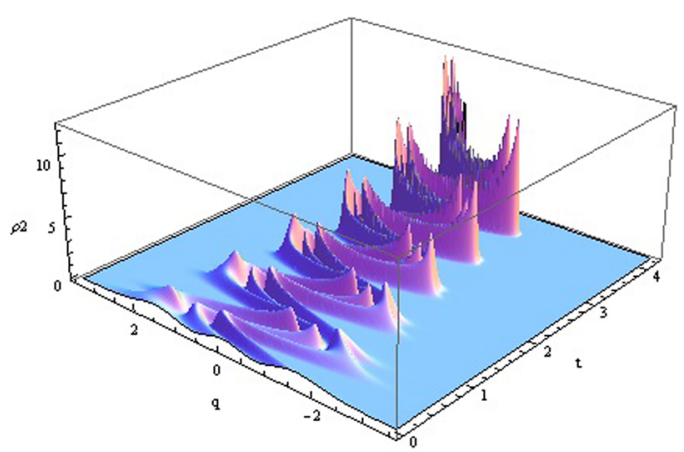

(b)

FIG. 19. Model 3: Probability density $\rho_{2}(q, t)$ with $\gamma=1, \omega_{0}=\sqrt{12}, \Lambda_{0}=\sqrt{209} / 2, \Omega_{d}=8$. (a) $\rho_{2}(q, t)$ with $x_{p}(t)=0$ and (b) $\rho_{2}(q, t)$ displaced by periodic $x_{p}(t)$ at resonance frequency $\omega=\sqrt{255} / 2, D_{0}=8, \hbar=1$.

\section{SUMMARY AND DISCUSSION}

We investigated the behavior of a Caldirola-Kanai quantum parametric oscillator under the influence of the mixed term $B(t)(\hat{q} \hat{p}+\hat{p} \hat{q}) / 2$ and linear terms $D_{0}(t) \hat{q}, E_{0}(t) \hat{p}$. The squeezing parameter $B(t)$ modifies the frequency of the corresponding classical equation and therefore has influence on the amplitude and squeezing properties of the wave packets. On the other hand, parameters $D_{0}(t)$ and $E_{0}(t)$ are responsible for the displacement of the wave packets. According to this, we introduced all possible choices of $B(t)$ so that the classical equation in position space preserves its damped harmonic oscillator structure with constant frequency and damping. Then, taking also sinusoidal external forces, squeezing effects and resonance phenomena were explicitly discussed according to the frequency modification. We found that for certain values of frequency modification, the generalized models show the same qualitative behavior as the standard Caldirola-Kanai oscillator, but for other values we observed rather unusual properties such as smooth probability densities with squeezing and breathing width, at the expense of singularities in momentum.

More precisely, to have a damped harmonic oscillator with constant frequency and damping in position space, we defined $B(t)$ leading to constant frequency modification $\Lambda_{0}^{2}$ so that the modified frequency $\Omega_{m}^{2}=\omega_{0}^{2}+\Lambda_{0}^{2}$ is always positive. Then, for $-\omega_{0}^{2}<\Lambda_{0}^{2}<\gamma^{2} / 4$, we discussed the model with constant $B(t)=B_{0}$ and Model 1 for which $B(t)=-(\gamma / 2)+\Omega_{B}^{\prime} \tanh \left(\Omega_{B}^{\prime} t-\beta\right), \Omega_{B}^{\prime}=\sqrt{\left(\gamma^{2} / 4\right)-\Lambda_{0}^{2}}$. We have seen that both of these models show essentially the same behavior as the standard CaldirolaKanai oscillator. However, the influence of $B(t)$ can be used to control squeezing and resonance and even change the type of the original oscillator as overdamped, critical or underdamped. In this work, 
we focused our attention on the cases when the damping is weak and forces are sinusoidal, but it is obvious that the results can be easily extended to critical and overdamping cases and different types of driving forces, when necessary.

More interesting situations appeared in Model 2 and Model 3. In Model 2, where $B(t)=-(\gamma / 2)$ $+b /(1+b t)$, the frequency modification is $\Lambda_{0}^{2}=\gamma^{2} / 4$, for any real $b$. In that case, the frequency of the modified oscillator $\Omega_{d}$ becomes equal to the natural frequency $\omega_{0}$, that is, modification cancels the effect of damping $\gamma$ in the damping frequency $\Omega_{d}$. Therefore, the frequency of squeezing is $\omega_{0}$ for all $b$, but the amplitude of oscillations can be increased by increasing the value of $|b|$. Moreover, for this model, the resonance frequency does not depend on $b$, and it is equal to the frequency of the damped oscillator when $B(t)=0$, that is, $\omega_{\text {res }}=\sqrt{\omega_{0}^{2}-\gamma^{2} / 4}=\Omega_{0}$.

Clearly Model 3, with $B(t)=-(\gamma / 2)-\Omega_{B} \tan \left(\Omega_{B} t-\beta\right), \Omega_{B}=\sqrt{\Lambda_{0}^{2}-\left(\gamma^{2} / 4\right)}$, is different comparing with the previous models. In the models with $B(t)=B_{0}$ and Model 1 , for given $\gamma$ and $\omega_{0}$, the set of allowed values of $\Lambda_{0}^{2}$ is bounded, and when the frequency increases, the amplitude of oscillations decreases and approaches the critical squeezing. On the other side, Model 3 is defined for $\Lambda_{0}^{2}>\gamma^{2} / 4$. Then, for given $\gamma$ and $\omega_{0}$, by increasing the value of $\Lambda_{0}^{2}$, one can simultaneously increase the wave amplitude and frequency of its oscillating width or get very high quality number $Q=\sqrt{\omega_{0}^{2}+\Lambda_{0}^{2}} / \gamma$. In this model, such properties were achieved by allowing singularities in momentum due to singularities in $B(t)$, when preserving the form of the classical equation of motion. Similar properties were discussed in Ref. 28, for generalized Hermite, Laguerre, and Jacobi type quantum parametric oscillators. Clearly, the study of quantum models with frequency modification without changing the structure of the oscillator can be extended to other special functions and equations. ${ }^{47}$ However, interesting problems can also appear when the frequency is essentially modified under the influence of the squeezing parameter $B(t)$. For example, it is possible to choose $B(t)$ so that the modified classical oscillator becomes of Mathieu type, for which the parametric resonance and its influence on the quantum problem can be investigated. The problems discussed here can also provide a basis for some other investigations on the subject, such as time-evolution of nonlinear Madelung fluids and pole dynamics, Schrödinger-Langevin formalism for describing interactions in non-conservative systems, and the multidimensional problems, the details of which are under consideration.

\section{ACKNOWLEDGMENTS}

This research is supported by The Scientific and Technological Research Council of Turkey, TÜBITAK, Project No. 116F206.

${ }^{1}$ R. K. Colegrave and M. S. Abdalla, Opt. Acta 28, 495 (1981).

${ }^{2}$ I. A. Pedrosa, A. Rosas, and I. Guedes, J. Phys. A: Math. Gen. 38, 7757 (2005).

${ }^{3}$ G. Dattoli, A. Torre, and J. C. Gallardo, Riv. Nuovo Cimento, Ser. 3 11, 1 (1998).

${ }^{4}$ A. B. Nassar, Phys. Lett. A 106, 43 (1984).

${ }^{5}$ A. Malkin, V. I. Manko, and D. A. Trifonov, Phys. Lett. A 30(7), 414 (1969).

${ }^{6}$ A. Sakharov, Zh. Eksp. Teor. Fiz. 49, 345 (1965) [Sov. Phys. JETP 22, 241 (1966)].

${ }^{7}$ W. P. Schleich and H. Walther, Elements of Quantum Information (Wiley-Verlag, Weinheim, 2007).

${ }^{8}$ M. S. Sarandy, E. I. Duzzioni, and R. M. Serra, Phys. Lett. A 375, 3343 (2011).

${ }^{9}$ P. Caldirola, Il Nouovo Cimento 18, 393 (1941).

${ }^{10}$ E. Kanai, Prog. Theor. Phys. 3, 440 (1948).

${ }^{11}$ H. Dekker, "Classical and quantum mechanics of damped harmonic oscillator," Phys. Rep. 80, 1-110 (1981).

${ }^{12}$ K. Husimi, Progr. Theor. Phys. 9(3), 238 (1953).

${ }^{13}$ R. Feynman, Phys. Rev. 84, 108 (1951).

${ }^{14}$ H. R. Lewis, Jr. and W. B. Riesenfeld, J. Math. Phys. 10, 1458 (1969).

15 J. Wei and E. Norman, J. Math. Phys. 4, 575 (1963).

${ }^{16}$ R. K. Colegrave and M. Sebawe Abdalla, J. Phys. A: Math. Gen. 14, 2269-2280 (1981).

${ }^{17}$ M. Sebawe Abdalla, Phys. Rev. A 33(5), 2870 (1986).

${ }^{18}$ V. V. Dodonov and V. I. Man'ko, Phys. Rev. A 20, 550 (1979).

19 A. Lopes de Lima, A. Rosas, and I. A. Pedrosa, Ann. Phys. 323, 2253-2264 (2008).

${ }^{20}$ D. C. Khandekar and S. V. Lawande, J. Math. Phys. 20(9), 1870 (1979).

${ }^{21}$ K. H. Yeon, D. H. Kim, C. I. Um, T. F. George, and L. N. Pandey, Phys. Rev. A 55(6), 4023 (1997).

${ }^{22}$ K. H. Yeon, D. H. Kim, S. Zhang, C. I. Um, S. K. Hong, and I. H. Kim, J. Korean Phys. Soc. 30(2), 506 (1997). 
23 J. R. Choi, Pramana-J. Phys. 62(1), 13 (2004).

${ }^{24}$ J. R. Choi and D.-W. Kim, J. Korean Phys. Soc. 45(6), 1426 (2004).

25 J. R. Choi and I. H. Nahm, Int. J. Theor. Phys. 46, 1 (2007).

${ }^{26}$ K. H. Yeon, C. I. Um, and T. F. George, Phys. Rev. A 68, 052108 (2003).

${ }^{27}$ D.-Y. Song, Phys. Rev. A 59(4), 2616 (1999).

${ }^{28}$ Ş. A. Büyükaşı and Z. Çayiç, J. Math. Phys. 57, 122107 (2016).

${ }^{29}$ E. Schrödinger, Naturwissenschaften 14, 664 (1926).

${ }^{30}$ R. J. Glauber, Phys. Rev. 131, 2766 (1963).

${ }^{31}$ J. R. Klauder, Ann. Phys. 11, 123 (1960).

${ }^{32}$ E. C. G. Sudarshan, Phys. Rev. Lett. 10, 227 (1963).

${ }^{33}$ M. M. Nieto, e-print arXiv:quant-ph/9708012v1 (1997).

${ }^{34}$ M. M. Nieto, Phys. Rev. Lett. 71, 2843-2846 (1993).

${ }^{35}$ V. V. Dodonov, J. Opt. B: Quantum Semiclassical Opt. 4(3), S98 (2002).

${ }^{36}$ V. V. Dodonov and V. I. Man'ko, Theory of Nonclassical States of Light (Taylor and Francis, 2003).

${ }^{37}$ I. A. Malkin and V. I. Manko, Dynamical Symmetries and Coherent States of Quantum Systems (Nauka, Moscow, 1979) (in Russian).

38 A. Perelomov, Generalized Coherent States and Their Applications (Springer-Verlag, 1986).

${ }^{39}$ H. P. Yuen, Phys. Rev. A 13(6), 2226-2243 (1976).

${ }^{40}$ M. Hillary, Phys. Rev. A 36(8), 3796-3802 (1987).

${ }^{41}$ D. F. Walls, "Squeezed states of light," Nature 306, 141-146 (1983).

${ }^{42}$ G. Breitenbach, S. Schiller, and J. Mlynek, Nature 387, 471-475 (1997).

${ }^{43}$ C. F. Lo, Phys. Rev. A 43(1), 404 (1991).

${ }^{44}$ C. F. Lo, Phys. Scr. 42, 389-392 (1990).

${ }^{45}$ C. F. Lo, Il Nuovo Cimento D 13, 127 (1991).

${ }^{46}$ S. K. Singh and S. Mandal, Opt. Commun. 283, 4685-4695 (2010).

${ }^{47}$ Ş. A. Büyükaşık, O. K. Pashaev, and E. Ulaş-Tigrak, J. Math. Phys. 50, 072102 (2009). 